\title{
Spectroscopy and Tautomerization Studies of Porphycenes
}

\author{
Jacek Waluk
}

Institute of Physical Chemistry, Polish Academy of Sciences, 01-224 Warsaw, Kasprzaka 44/52, Poland;

Faculty of Mathematics and Science, Cardinal Stefan Wyszyński University, Dewajtis 5, 01-815 Warsaw, Poland

\author{
E-mail:waluk@ichf.edu.pl
}

\section{Supporting Information}

\section{Table of contents}

\section{Table S1}

Table S2

Table S3

References 
Table S1. Absorption data for free base porphycenes: wavelengths corresponding to the maxima of the lowest energy $\left(\lambda\left(\mathrm{S}_{1}\right)\right)$ and Soret $\left(\lambda\left(\mathrm{S}_{\text {oret }}\right)\right)$ transitions and the corresponding absorption coefficients $(\varepsilon)$.

\begin{tabular}{|c|c|c|c|c|c|c|}
\hline Compound & Solvent & $\begin{array}{l}\lambda\left(\mathrm{S}_{1}\right) \\
{[\mathrm{nm}]}\end{array}$ & $\varepsilon / 10^{4}$ & $\begin{array}{c}\lambda \text { (Soret) } \\
{[\mathrm{nm}]}\end{array}$ & $\begin{array}{c}\varepsilon / 10^{4} \\
{\left[\mathrm{M}^{-1} \mathrm{~cm}^{-1}\right\rceil}\end{array}$ & Reference \\
\hline
\end{tabular}

Alkyl-substituted porphycenes

\begin{tabular}{|c|c|c|c|c|c|c|}
\hline \multirow[t]{4}{*}{1} & Benzene & 630 & 5.2 & 358 & 13.9 & 1 \\
\hline & Toluene & 633 & 4.2 & 362 & 10.6 & 2 \\
\hline & $\mathrm{CH}_{2} \mathrm{Cl}_{2}$ & 629 & & & & 2 \\
\hline & $\mathrm{MeOH}$ & 627 & & & & 2 \\
\hline 11 & $\mathrm{CH}_{2} \mathrm{Cl}_{2}$ & 631 & 4.1 & 368 & 14.1 & 3 \\
\hline 12 & $\mathrm{CH}_{2} \mathrm{Cl}_{2}$ & 632 & 4.8 & 370 & 14.2 & 3 \\
\hline \multirow[t]{3}{*}{13} & Toluene & 637 & 5.0 & 372 & 13.9 & 2 \\
\hline & $\mathrm{CH}_{2} \mathrm{Cl}_{2}$ & 633 & 4.8 & 370 & 14.2 & 3 \\
\hline & THF & 635 & 4.0 & & & \\
\hline 14 & $\mathrm{CH}_{2} \mathrm{Cl}_{2}$ & 633 & 4.7 & 371 & 14.5 & 5 \\
\hline 15 & $\mathrm{CH}_{2} \mathrm{Cl}_{2}$ & 632 & 4.9 & 370 & 14.7 & 6 \\
\hline 16 & Acetonitrile & 628 & & 358 & & 7 \\
\hline 17 & Acetonitrile & 628 & & 362 & & 7 \\
\hline 18 & Acetonitrile & 630 & & 364 & & 7 \\
\hline \multirow[t]{3}{*}{19} & $\mathrm{CH}_{2} \mathrm{Cl}_{2}$ & 665 & 3.1 & 385 & 14.4 & 8 \\
\hline & EtOH & 664 & 2.2 & 382 & 10.2 & 9 \\
\hline & $\mathrm{CH}_{2} \mathrm{Cl}_{2}$ & 665 & 3.0 & 385 & 14.5 & 120 \\
\hline 20 & $\mathrm{CH}_{2} \mathrm{Cl}_{2}$ & 657 & 3.0 & 382 & 14.4 & 8,10 \\
\hline 21 & $\mathrm{CH}_{2} \mathrm{Cl}_{2}$ & 720 & 4.3 & 376 & 8.9 & 11 \\
\hline 22 & $\mathrm{CH}_{2} \mathrm{Cl}_{2}$ & 694 & & 407 & & 12 \\
\hline 23 & $\mathrm{CH}_{2} \mathrm{Cl}_{2}$ & 657 & 4.3 & 369 & 14.1 & 13 \\
\hline 24 & $\mathrm{CH}_{2} \mathrm{Cl}_{2}$ & 658 & 5.0 & 372 & 17.0 & 13 \\
\hline 25 & $\mathrm{CH}_{2} \mathrm{Cl}_{2}$ & 659 & 4.6 & 373 & 16.1 & 13 \\
\hline 26 & $\mathrm{CH}_{2} \mathrm{Cl}_{2}$ & 647 & 3.4 & 381 & 12.6 & 14 \\
\hline 27 & Acetonitrile & 645 & 2.9 & 362 & 9.4 & 15 \\
\hline 28 & Acetonitrile & 645 & 3.6 & 368 & 12.4 & 15 \\
\hline 29 & $\mathrm{CH}_{2} \mathrm{Cl}_{2}$ & 646 & 2.9 & 383 & 12.9 & 5 \\
\hline 30 & $\mathrm{CH}_{2} \mathrm{Cl}_{2}$ & 706 & 3.4 & 381 & 11.8 & 14 \\
\hline 31 & $\mathrm{CH}_{2} \mathrm{Cl}_{2}$ & 707 & 3.3 & 385 & 10.5 & 14 \\
\hline 32 & $\mathrm{CH}_{2} \mathrm{Cl}_{2}$ & 652 & 3.5 & 386 & 13.8 & 16 \\
\hline 33 & $\mathrm{CH}_{2} \mathrm{Cl}_{2}$ & 639 & 4.0 & 372 & 13.8 & 5 \\
\hline 34 & $\mathrm{CH}_{2} \mathrm{Cl}_{2}$ & 642 & 4.3 & 373 & 13.5 & 16 \\
\hline \multirow[t]{2}{*}{35} & Toluene & 677 & 2.4 & & & 17 \\
\hline & $\mathrm{CH}_{2} \mathrm{Cl}_{2}$ & 673 & 1.8 & 369 & 9.8 & 18 \\
\hline 36 & Toluene & 682 & 1.7 & & & 17 \\
\hline 37 & Toluene & 677 & 1.7 & & & 17 \\
\hline 38 & $\mathrm{CH}_{2} \mathrm{Cl}_{2}$ & 641 & 3.1 & 372 & 14.3 & 18,19 \\
\hline
\end{tabular}




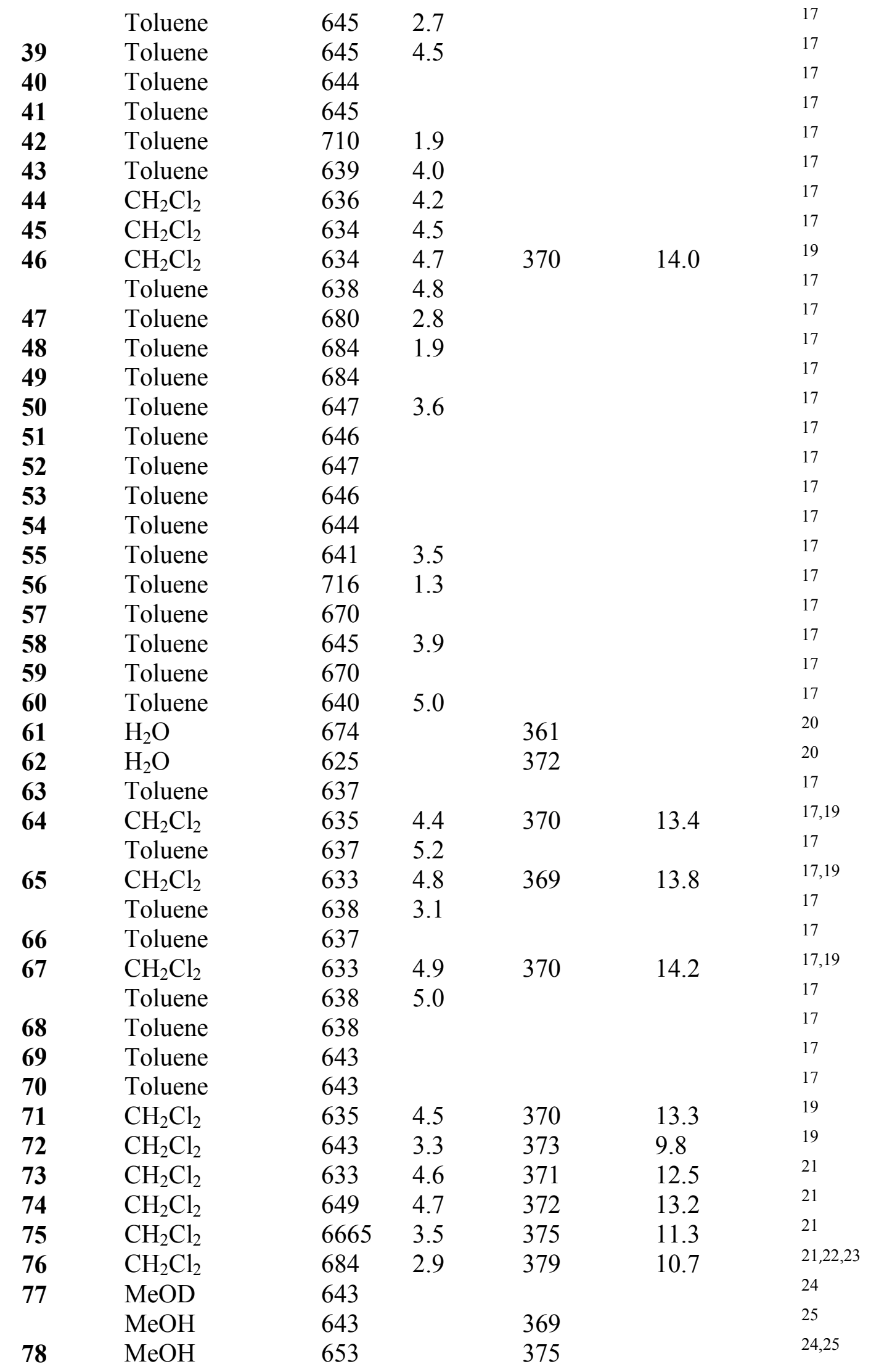




\begin{tabular}{|c|c|c|c|c|c|c|}
\hline \multirow{5}{*}{79} & MeOD & \multicolumn{2}{|l|}{653} & \multirow[b]{2}{*}{374} & & 25 \\
\hline & $\mathrm{H}_{2} \mathrm{O}$ & 646 & & & & 24 \\
\hline & $\mathrm{MeOH}$ & 653 & & 375 & & 25 \\
\hline & MeOD & 653 & & & & 24 \\
\hline & $\mathrm{H}_{2} \mathrm{O}$ & 646 & & 374 & & 25 \\
\hline 80 & $\mathrm{MeOH}$ & 688 & & 372 & & 25 \\
\hline & MeOD & 688 & & & & 24 \\
\hline & $\mathrm{H}_{2} \mathrm{O}$ & 686 & & 371 & & 25 \\
\hline 81 & $\mathrm{CH}_{2} \mathrm{Cl}_{2}$ & 652 & & 372 & & 26 \\
\hline & $\begin{array}{l}\text { Sol-gel silica } \\
\text { film }\end{array}$ & 651 & & 375 & & 26 \\
\hline 82 & $\mathrm{CH}_{2} \mathrm{Cl}_{2}$ & 656 & & 375 & & 27 \\
\hline 83 & Ionic liquid & 651 & 3.8 & 373 & 9.5 & 27 \\
\hline 84 & $\mathrm{CH}_{2} \mathrm{Cl}_{2}$ & 656 & 3.9 & 376 & 10.7 & 28 \\
\hline 85 & $\mathrm{CH}_{2} \mathrm{Cl}_{2}$ & 654 & 3.5 & 375 & 10.4 & 28 \\
\hline 86 & Acetone & 668 & & 391 & & 7 \\
\hline 87 & Acetone & 665 & & 390 & & 7 \\
\hline 88 & $\mathrm{CHCl}_{3}$ & 644 & 4.3 & 374 & 13.1 & 29 \\
\hline 89 & $\mathrm{CHCl}_{3}$ & 652 & 4.4 & 386 & 13.1 & 29 \\
\hline 90 & $\mathrm{CHCl}_{3}$ & 646 & 4.7 & 375 & 13.1 & 29 \\
\hline 91 & $\mathrm{CHCl}_{3}$ & 647 & 0.91 & 376 & 25.8 & 29 \\
\hline 92 & $\mathrm{CHCl}_{3}$ & 658 & 4.6 & 376 & 12.6 & 29 \\
\hline 93 & $\mathrm{CHCl}_{3}$ & 661 & 4.2 & 391 & 12.8 & 29 \\
\hline 94 & $\mathrm{CH}_{2} \mathrm{Cl}_{2}$ & 654 & 3.9 & 378 & 12.0 & 29 \\
\hline 95 & $\mathrm{CH}_{2} \mathrm{Cl}_{2}$ & 650 & 3.9 & 391 & 13.3 & 29 \\
\hline 96 & $\mathrm{CHCl}_{3}$ & 647 & 10.0 & 373 & 22.9 & 29 \\
\hline 97 & $\mathrm{CH}_{2} \mathrm{Cl}_{2}$ & 650 & 10.2 & 377,394 & $21.9,21.9$ & 30 \\
\hline 98 & $\mathrm{CHCl}_{3}$ & 648 & 7.4 & 373 & 11.5 & 29 \\
\hline 99 & $\mathrm{CHCl}_{3}$ & 654 & 17.7 & 374 & 23.8 & 29 \\
\hline 100 & $\mathrm{CHCl}_{3}$ & 656 & 14.0 & 374 & 10.9 & 29 \\
\hline 101 & THF & 638 & & 376 & & 31 \\
\hline 102 & $\mathrm{THF}$ & 637 & 2.7 & 376 & 9.5 & 31 \\
\hline 103 & $\mathrm{CH}_{2} \mathrm{Cl}_{2}$ & 632 & 3.4 & 373 & 15.5 & 32 \\
\hline 104 & $\mathrm{CH}_{2} \mathrm{Cl}_{2}$ & 632 & 3.0 & 372 & 13.8 & 32 \\
\hline 105 & $\mathrm{H}_{2} \mathrm{O}$ & $\sim 630$ & & 372 & & 33 \\
\hline 106 & $\mathrm{CH}_{2} \mathrm{Cl}_{2}$ & 640 & & 373 & & 34 \\
\hline 107 & $\mathrm{CH}_{2} \mathrm{Cl}_{2}$ & 640 & & 372 & & 34 \\
\hline 108 & $\mathrm{CH}_{2} \mathrm{Cl}_{2}$ & 640 & & 372 & & 34 \\
\hline 109 & $\mathrm{CH}_{2} \mathrm{Cl}_{2}$ & 648 & & 373 & & 34 \\
\hline 110 & $\mathrm{CH}_{2} \mathrm{Cl}_{2}$ & 645 & & 373 & & 34 \\
\hline 111 & $\mathrm{CH}_{2} \mathrm{Cl}_{2}$ & 639 & 3.3 & 375 & 9.9 & 35 \\
\hline 112 & $\mathrm{CH}_{2} \mathrm{Cl}_{2}$ & 716 & 1.7 & 369 & 9.7 & 35 \\
\hline 113 & $\mathrm{CH}_{2} \mathrm{Cl}_{2}$ & 636 & & 375 & & 35 \\
\hline 114 & $\mathrm{CH}_{2} \mathrm{Cl}_{2}$ & 697 & & 372 & & 35 \\
\hline 115 & $\mathrm{CHCl}_{3}$ & 647 & 3.3 & 376 & 14.5 & 36 \\
\hline 116 & $\mathrm{CHCl}_{3}$ & 664 & 2.9 & 385 & 13.5 & 36 \\
\hline
\end{tabular}




$\begin{array}{lllllll}\mathbf{1 1 7} & \mathrm{CHCl}_{3} & 636 & 2.2 & 382 & 12.3 & 37 \\ \mathbf{1 1 8} & \mathrm{CHCl}_{3} & 699 & 5.0 & 411 & 7.2 & 38 \\ \mathbf{1 1 9} & \mathrm{CH}_{2} \mathrm{Cl}_{2} & 618 & 5.7 & 380 & 13.0 & 39 \\ \mathbf{1 2 0} & \mathrm{CH}_{2} \mathrm{Cl}_{2} & 680 & 2.6 & 387 & 7.8 & 40 \\ \mathbf{1 2 1} & \mathrm{CH}_{2} \mathrm{Cl}_{2} & 640 & 3.2 & 387 & 9.3 & 40\end{array}$

\section{Aryl-substituted porphycenes}

\begin{tabular}{|c|c|c|c|c|c|}
\hline \multirow[t]{2}{*}{122} & Toluene & 659 & 5.0 & 378 & 11.8 \\
\hline & $\mathrm{THF}$ & 656 & 4.2 & & \\
\hline 122 & Benzene & 658 & 3.9 & 377 & 8.9 \\
\hline 122 & $\mathrm{CHCl}_{3}$ & 657 & 3.7 & 377 & 8.7 \\
\hline 122 & Pyridine & 658 & 3.8 & 379 & 8.7 \\
\hline 122 & DMF & 655 & 3.8 & 377 & 8.5 \\
\hline 122 & DMSO & 656 & 3.3 & 378 & 7.4 \\
\hline 122 & $\mathrm{AcOH}$ & 654 & & 377 & \\
\hline 122 & $\mathrm{CF}_{3} \mathrm{COOH}$ & 648 & 5.0 & $383 / 422$ & $7.8 / 7.8$ \\
\hline 122 & $\mathrm{H}_{2} \mathrm{SO}_{4}$ & 655 & 5.3 & $385 / 429$ & $8.5 / 8.5$ \\
\hline 123 & Toluene & 656 & 5.4 & 376 & 15.1 \\
\hline 124 & Toluene & 657 & 5.6 & 400 & 13.3 \\
\hline 125 & Toluene & 763 & 2.1 & 375 & 9.3 \\
\hline 126 & Toluene & 659 & 4.4 & 378 & 10.0 \\
\hline \multirow[t]{2}{*}{127} & Toluene & 701 & 2.5 & 384 & 13.1 \\
\hline & $\mathrm{CH}_{2} \mathrm{Cl}_{2}$ & 696 & 2.0 & 381 & 13.6 \\
\hline 128 & $\mathrm{PBS}^{\mathrm{a}}$ & 655 & & 375 & \\
\hline 129 & THF & 660 & 3.7 & 377 & 9.4 \\
\hline 130 & $\mathrm{CH}_{2} \mathrm{Cl}_{2}$ & 662 & 4.7 & 380 & 10.4 \\
\hline 131 & $\mathrm{CH}_{2} \mathrm{Cl}_{2}$ & 658 & 5.4 & 376 & 12.2 \\
\hline 132 & $\mathrm{CHCl}_{3}$ & 679 & 2.1 & 413 & 9.2 \\
\hline 133 & $\mathrm{CHCL}_{3}$ & 679 & 2.1 & 413 & 8.3 \\
\hline 134 & $\mathrm{THF}$ & 659 & 6.9 & 373 & 15.6 \\
\hline 135 & benzene & 659 & 5.8 & 378 & 13.1 \\
\hline 136 & toluene & 666 & 4.6 & 380 & 10.2 \\
\hline 137 & $\mathrm{MeOH}$ & 656 & 11.2 & 375 & 23.2 \\
\hline \multirow[t]{2}{*}{138} & $\mathrm{MeOH}$ & 657 & 4.7 & 377 & 10.2 \\
\hline & Water & 641 & 2.0 & 366 & 7.2 \\
\hline 139 & $\mathrm{CH}_{2} \mathrm{Cl}_{2}$ & 660 & 3.73 & 377 & 9.8 \\
\hline 140 & $\mathrm{CH}_{2} \mathrm{Cl}_{2}$ & 649 & 4.1 & 376 & 11.2 \\
\hline 141 & $\mathrm{CH}_{2} \mathrm{Cl}_{2}$ & 653 & 3.4 & 378 & 7.5 \\
\hline 142 & Acetone & 667 & 2.1 & 399 & 8.8 \\
\hline 143 & Acetone & 745 & 0.93 & 390 & 10.1 \\
\hline 144 & Acetone & 743 & 2.0 & 394 & 10.6 \\
\hline 145 & Acetone & 763 & 1.4 & 387 & 9.6 \\
\hline 146 & Acetone & 744 & 1.8 & 392 & 10.5 \\
\hline 147 & Acetone & 740 & 1.2 & 398 & 0.58 \\
\hline 148 & Acetone & 750 & 1.2 & 386 & 0.76 \\
\hline
\end{tabular}




$\begin{array}{lllllll}\mathbf{1 4 9} & \text { Acetone } & 748 & 1.7 & 388 & 10.0 & 52 \\ \mathbf{1 5 0} & \mathrm{CH}_{2} \mathrm{Cl}_{2} & 653 & 4.3 & 382 & 15.3 & 53 \\ & \mathrm{CHCl}_{3} & 653 & 2.5 & 381 & 12.6 & 54 \\ \mathbf{1 5 1} & \mathrm{CH}_{2} \mathrm{Cl}_{2} & 655 & 3.8 & 383 & 12.3 & 53 \\ & \mathrm{CHCl}_{3} & 654 & 3.2 & 383 & 10.0 & 54 \\ \mathbf{1 5 2} & \mathrm{CHCl}_{3} & 655 & 3.2 & 382 & 10.0 & 54 \\ \mathbf{1 5 3} & \mathrm{CHCl}_{3} & 650 & 2.5 & 380 & 7.9 & 54 \\ \mathbf{1 5 4} & \mathrm{CHCl}_{3} & 653 & 2.0 & 379 & 7.9 & 54 \\ \mathbf{1 5 5} & \mathrm{CHCl}_{3} & 650 & 2.5 & 380 & 10.0 & 54 \\ \mathbf{1 5 6} & \mathrm{CH}_{2} \mathrm{Cl}_{2} & 661 & 3.5 & 419 & 8.8 & 55\end{array}$

Heteroatom-containing porphycene analogs

$\begin{array}{lllllll}\mathbf{1 5 7} & \mathrm{CH}_{2} \mathrm{Cl}_{2} & 686 & 7.7 & 373 & 13.1 & 56 \\ \mathbf{1 5 8} & \text { Toluene } & 760 & 2.0 & 388 & 3.4 & 57,58 \\ \mathbf{1 5 9} & \text { Benzene } & 752 & & 383 & & 58 \\ \mathbf{1 6 0} & \mathrm{MeOH} & 758 & 1.7 & 388 & 3.7 & 58 \\ \mathbf{1 6 1} & \mathrm{THF} & 717 & 1.78 & 387 & 8.1 & 59 \\ \mathbf{1 6 2} & \mathrm{THF} & 740 & 0.56 & 388 & 5.9 & 60 \\ \mathbf{1 6 3} & \mathrm{THF} & 758 & 1.7 & 398 & 6.5 & 60 \\ \mathbf{1 6 4} & \mathrm{THF} & 776 & 1.6 & 410 & 5.6 & 60 \\ \mathbf{1 6 5} & 70 \% \mathrm{HClO}_{4} & 599 & 4.5 & 376 & 38.3 & 61 \\ & \mathrm{H}_{2} \mathrm{SO}_{4} & 599 & 2.3 & 382 & 35.0 & 62\end{array}$

\section{Benzoporphycenes and Naphthoporphycenes}

$\begin{array}{lllllll}\mathbf{1 6 6} & \mathrm{CH}_{2} \mathrm{Cl}_{2} & 639 & 4.2 & 396 & 10.1 & 19 \\ \mathbf{1 6 7} & \mathrm{CH}_{2} \mathrm{Cl}_{2} & 636 & 3.6 & 396 & 8.9 & 19 \\ \mathbf{1 6 8} & \mathrm{CH}_{2} \mathrm{Cl}_{2} / \mathrm{IPA} & 629 & 0.64 & 407 & 2.9 & 12 \\ \mathbf{1 6 9} & n \text {-Hexane } & 764 & 1.7 & 375 & 3.1 & 63 \\ & \text { Acetonitrile } & 750 & 1.7 & 371 & 3.3 & 63 \\ & \text { THF } & 763 & 1.8 & 373 & 2.4 & 63 \\ & \text { Toluene } & 764 & 2.2 & & & 17 \\ \mathbf{1 7 0} & n \text {-Hexane } & 765 & 2.1 & 375 & 3.7 & 63 \\ & \text { Acetonitrile } & 749 & 2.1 & 371 & 4.1 & 63 \\ \mathbf{1 7 1} & \mathrm{PrOH}_{\mathbf{1 7 2}} & 757 & 2.0 & 374 & 4.1 & 63 \\ \mathbf{1 7 3} & \mathrm{CH}_{2} \mathrm{Cl}_{2} & 647 & 6.2 & 410 & 12.9 & 5 \\ \mathbf{1 7 4} & \mathrm{CH}_{2} \mathrm{Cl}_{2} & 663 & 2.9 & 390 & 10.7 & 55 \\ \mathbf{1 7 5} & \mathrm{CH}_{2} \mathrm{Cl}_{2} & 670 & 3.6 & 434 & 27.5 & 5 \\ \mathbf{1 7 6} & \mathrm{CH}_{2} \mathrm{Cl}_{2} & 733 & 3.6 & 448 & 15.9 & 14 \\ \mathbf{1 7 7} & \mathrm{CH}_{2} \mathrm{Cl}_{2} & 741 & 3.0 & 450 & 13.2 & 14 \\ & \mathrm{THF}_{178} & 659 & 10.0 & 462 & 14.1 & 16 \\ & \mathrm{Toluene} & 717 & & 496 & & \text { This work } \\ & \mathrm{CH}_{2} \mathrm{Cl}_{2} & 718 & & 499 & & \text { This work } \\ & \mathrm{CH}_{2} \mathrm{Cl}_{2} & 717 & 1.7 & 401 & 3.5 & 65\end{array}$




\begin{tabular}{lllllll}
$\mathbf{1 7 9}$ & $\mathrm{CH}_{2} \mathrm{Cl}_{2}$ & 725 & - & 404 & - & 66 \\
$\mathbf{1 8 0}$ & $\mathrm{CH}_{2} \mathrm{Cl}_{2}$ & 713 & 3.4 & 400 & 7.1 & 65 \\
$\mathbf{1 8 1}$ & $\mathrm{CH}_{2} \mathrm{Cl}_{2}$ & 714 & 4.2 & 400 & 8.8 & 65 \\
$\mathbf{1 8 2}$ & $\mathrm{CH}_{2} \mathrm{Cl}_{2}$ & 726 & 2.6 & 399 & 4.0 & 64 \\
$\mathbf{1 8 3}$ & $\mathrm{CH}_{2} \mathrm{Cl}_{2}$ & 734 & 2.2 & 378 & 4.1 & 64 \\
$\mathbf{1 8 4}$ & $\mathrm{CH}_{2} \mathrm{Cl}_{2}$ & 727 & 1.1 & 404 & 2.4 & 64 \\
$\mathbf{1 8 5}$ & $\mathrm{CH}_{2} \mathrm{Cl}_{2}$ & 721 & 3.4 & 388 & 21.0 & 67 \\
$\mathbf{1 8 6}$ & $\mathrm{Tolluene}_{\mathbf{1 8 7}}$ & 762 & 2.3 & & & 17 \\
\hline
\end{tabular}

${ }^{\mathrm{a}}$ Phosphate-buffered saline 
Table S2. Absorption data for metalloporphycenes: wavelengths corresponding to the maxima of the lowest energy $\left(\lambda\left(\mathrm{S}_{1}\right)\right)$ and Soret $\left(\lambda\left(\mathrm{S}_{\text {oret }}\right)\right)$ transitions and the corresponding absorption coefficients $(\varepsilon)$.

\begin{tabular}{|c|c|c|c|c|c|c|}
\hline Compound & Solvent & $\begin{array}{c}\lambda\left(\mathrm{S}_{1}\right) \\
{[\mathrm{nm}]}\end{array}$ & $\varepsilon / 10^{4}$ & $\begin{array}{c}\lambda \text { (Soret) } \\
{[\mathrm{nm}]}\end{array}$ & $\varepsilon / 10^{4}$ & Reference \\
\hline $1-\mathrm{SnCl}_{2}$ & 2-MTHF & 628 & 0.26 & 385 & 0.35 & 62 \\
\hline \multirow[t]{5}{*}{$13-\mathrm{Ni}$} & $\mathrm{CH}_{2} \mathrm{Cl}_{2}$ & 614 & 4.2 & 387 & 14.9 & 3 \\
\hline & $n$-Hexane & 617 & & 386 & & 62 \\
\hline & 2-MTHF & 612 & 4.6 & 386 & & 62 \\
\hline & Acetonitrile & 617 & & 403 & 12.6 & 62 \\
\hline & $\mathrm{CH}_{2} \mathrm{Cl}_{2}$ & 601 & 3.2 & 388 & 6.7 & 68 \\
\hline \multirow[t]{4}{*}{ 13-Cu } & $\mathrm{CH}_{2} \mathrm{Cl}_{2}$ & 613 & 7.0 & 387 & 15.4 & $23,69,70$ \\
\hline & Acetonitrile & 613 & & 383 & & 71 \\
\hline & $\mathrm{CH}_{2} \mathrm{Cl}_{2}$ & 614 & 4.3 & 386 & 8.7 & 68 \\
\hline & $\mathrm{CH}_{2} \mathrm{Cl}_{2}$ & 613 & 8.1 & 386 & 17.4 & 120 \\
\hline \multirow[t]{2}{*}{ 13-Pd } & Benzene & 615 & 3.4 & 386 & 6.9 & 72 \\
\hline & Benzene & 615 & 6.9 & 386 & 14.1 & 120 \\
\hline \multirow[t]{3}{*}{ 13-Pt } & Benzene & 590 & 4.8 & 380 & 9.9 & 72 \\
\hline & $\mathrm{CHCl}_{3}$ & 593 & 3.6 & 382 & 7.9 & 73 \\
\hline & Benzene & 590 & 4.8 & 380 & 10.0 & 120 \\
\hline \multirow[t]{3}{*}{ 13-Co } & THF/TBAP & $\sim 590$ & & $\sim 390$ & & 74 \\
\hline & $\mathrm{CH}_{2} \mathrm{Cl}_{2}$ & 594 & & 395 & & 75 \\
\hline & $\mathrm{CH}_{2} \mathrm{Cl}_{2}$ & 592 & 6.2 & 384 & 8.3 & 120 \\
\hline $13-\mathrm{CoO}_{2}$ & $\mathrm{CH}_{2} \mathrm{Cl}_{2}$ & 613 & & 390 & & 75 \\
\hline $13-\mathrm{CoCH}_{3}$ & $\mathrm{CH}_{2} \mathrm{Cl}_{2}$ & 592 & 4.0 & 385 & 9.2 & 76,77 \\
\hline $13-\mathrm{CoOOCH}_{3}$ & $\mathrm{CH}_{2} \mathrm{Cl}_{2}$ & 613 & & 385 & & 77 \\
\hline 13-CoCl & Toluene & 621 & & 396 & & 78 \\
\hline $13-$ & $\mathrm{CH}_{2} \mathrm{Cl}_{2}$ & 591 & & 384 & & 78 \\
\hline \multicolumn{7}{|l|}{$\mathrm{Co}\left(\mathrm{CH}_{2} \mathrm{CH}(\mathrm{OEt})_{2}\right)$} \\
\hline $13-\mathrm{CoPF}_{6}$ & $\mathrm{CH}_{2} \mathrm{Cl}_{2}$ & 604 & & 378 & & 79 \\
\hline \multirow[t]{2}{*}{ 13-FeCl } & THF/TBAP & $\sim 620$ & & $\sim 380$ & & 74 \\
\hline & $\mathrm{CH}_{2} \mathrm{Cl}_{2}$ & 615 & 5.1 & 362 & 9.3 & 120 \\
\hline $13-\mathrm{FeOCOCF}_{3}$ & $\mathrm{CH}_{2} \mathrm{Cl}_{2}$ & 613 & 4.5 & 366 & 6.5 & 120 \\
\hline$(13-\mathrm{Fe})_{2} \mathrm{O}$ & $\mathrm{CH}_{2} \mathrm{Cl}_{2}$ & 632 & 5.5 & 367 & 10.0 & 120 \\
\hline 13-AICI & $\mathrm{CH}_{2} \mathrm{Cl}_{2}$ & 610 & 12.3 & 390 & 21.9 & 120 \\
\hline 13-MnCl & $\mathrm{CH}_{2} \mathrm{Cl}_{2}$ & 626 & 3.1 & 341 & 5.4 & 120 \\
\hline $13-\operatorname{Ru}(\mathrm{cpy})_{2}{ }^{\mathrm{a}}$ & Benzene & 616 & 8.2 & 334 & 5.1 & 80 \\
\hline 13-Ru(CO)(py) & Benzene & 611 & 4.7 & 383 & 8.8 & 80,81 \\
\hline $13-R u(p y)_{2}$ & Benzene & 621 & 7.0 & $340 / 380$ & $6.1 / 4.1$ & 80 \\
\hline $13-\mathrm{Ru}(\mathrm{py})_{2} \mathrm{ClO}_{4}$ & $\mathrm{CH}_{2} \mathrm{Cl}_{2}$ & 607 & 2.1 & 374 & 7.9 & 80 \\
\hline $13-\mathrm{Ru}(\mathrm{dmap})_{2} \mathrm{ClO}_{4}{ }^{\mathrm{b}}$ & $\mathrm{CH}_{2} \mathrm{Cl}_{2}$ & 607 & 2.7 & 375 & 5.5 & 80 \\
\hline \multirow[t]{2}{*}{$13-\mathrm{SnCl}_{2}$} & $\mathrm{CH}_{2} \mathrm{Cl}_{2}$ & 625 & 8.9 & 391 & 13.4 & 82,83 \\
\hline & $\mathrm{CH}_{2} \mathrm{Cl}_{2}$ & 625 & 9.1 & 391 & 13.8 & 120 \\
\hline $13-\mathrm{SnF}_{2}$ & $\mathrm{CH}_{2} \mathrm{Cl}_{2}$ & 620 & 10.3 & 388 & 16.5 & 83 \\
\hline $13-\mathrm{SnBr}_{2}$ & $\mathrm{CH}_{2} \mathrm{Cl}_{2}$ & 627 & 7.8 & 393 & 12.5 & 83 \\
\hline
\end{tabular}




\begin{tabular}{|c|c|c|c|c|c|c|}
\hline 14-Ni & $\mathrm{CH}_{2} \mathrm{Cl}_{2}$ & 603 & 6.1 & 390 & 13.1 & 5 \\
\hline \multirow{5}{*}{$19-Z n$} & $\mathrm{EtOH}$ & 642 & 8.8 & 390 & 14.9 & 9 \\
\hline & $\mathrm{CH}_{2} \mathrm{Cl}_{2}$ & 638 & 9.5 & 393 & 16.1 & 8 \\
\hline & Acetonitrile & 636 & & 391 & & 71 \\
\hline & Toluene & 636 & & 394 & & 84 \\
\hline & $\mathrm{CH}_{2} \mathrm{Cl}_{2}$ & 638 & 9.5 & 393 & 16.2 & 120 \\
\hline 19-Zn(py) & $\mathrm{CH}_{2} \mathrm{Cl}_{2}$ & 644 & 9.2 & 395 & 14.6 & 8 \\
\hline 19-Zn(py) & Toluene & 647 & & 396 & & 84 \\
\hline $19-\operatorname{Zn}(\mathbf{p i})^{\mathrm{c}}$ & Toluene & 646 & & 394 & & 84 \\
\hline $19-\mathrm{ZnCl}_{2} \mathrm{Ph}$ & Toluene & 646 & & 394 & & 84 \\
\hline 19-ZnCIPh & Toluene & 647 & & 395 & & 84 \\
\hline 19-ZnMePh & Toluene & 647 & & 396 & & 84 \\
\hline 19-Co & $\mathrm{CH}_{2} \mathrm{Cl}_{2}$ & 599 & 4.5 & 387 & 9.1 & 85 \\
\hline 19-CoCl & $\mathrm{CH}_{2} \mathrm{Cl}_{2}$ & 618 & 2.3 & 391 & 6.2 & 76 \\
\hline $\mathrm{NCH}_{3}-19-\mathrm{CoCl}$ & $\mathrm{CH}_{2} \mathrm{Cl}_{2}$ & 666 & 2.0 & 402 & 3.9 & 76 \\
\hline $19-\mathrm{CoCH}_{3}$ & $\mathrm{CH}_{2} \mathrm{Cl}_{2}$ & 671 & 2.9 & 389 & 8.1 & 76 \\
\hline 19-Co(Ph) & $\mathrm{CH}_{2} \mathrm{Cl}_{2}$ & 612 & 2.0 & 390 & 7.1 & 76 \\
\hline \multirow[t]{2}{*}{ 19-Cu } & $\mathrm{CH}_{2} \mathrm{Cl}_{2}$ & 623 & 6.4 & 389 & 18.0 & 85 \\
\hline & $\mathrm{CH}_{2} \mathrm{Cl}_{2}$ & 622 & 3.1 & 385 & 9.5 & 68 \\
\hline \multirow[t]{3}{*}{ 19-Ni } & $\mathrm{CH}_{2} \mathrm{Cl}_{2}$ & 614 & 3.5 & 390 & 11.1 & $12,68,85$ \\
\hline & $\mathrm{CH}_{2} \mathrm{Cl}_{2}$ & 615 & 1.7 & 391 & 5.0 & 68 \\
\hline & $\mathrm{CH}_{2} \mathrm{Cl}_{2}$ & 614 & 3.5 & 390 & 11.0 & 120 \\
\hline 19-Mg & Toluene & 649 & 7.0 & 392 & 11.2 & 86 \\
\hline 19-Pd & $\mathrm{CHCl}_{3}$ & 618 & 4.1 & 390 & 9.8 & 37 \\
\hline 19-FeCl & $\mathrm{CH}_{2} \mathrm{Cl}_{2}$ & 622 & 3.7 & 368 & 8.9 & 85 \\
\hline 19-MnCl & $\mathrm{CH}_{2} \mathrm{Cl}_{2}$ & 633 & 2.8 & 348 & 5.8 & 85 \\
\hline 19-MoCl & Benzene & 648 & 2.5 & & & 87 \\
\hline 19-SnCl 2 & $\mathrm{CH}_{2} \mathrm{Cl}_{2}$ & 626 & 4.6 & 400 & 14.1 & 82 \\
\hline $19-\operatorname{Sn}(\mathrm{OH})_{2}$ & $\mathrm{CH}_{2} \mathrm{Cl}_{2}$ & 628 & 4.8 & 396 & 15.2 & 88 \\
\hline $19-\mathrm{Sn}(\mathrm{FcCOO})_{2}$ & $\mathrm{CH}_{2} \mathrm{Cl}_{2}$ & 628 & 5.9 & 397 & 19.2 & 88 \\
\hline 19-Ru(CO)(py) & Benzene & & & 385 & & 81 \\
\hline \multirow[t]{2}{*}{$20-\mathrm{Ni}$} & $\mathrm{CH}_{2} \mathrm{Cl}_{2}$ & 600 & 4.3 & 389 & 13.8 & 89 \\
\hline & $\mathrm{CH}_{2} \mathrm{Cl}_{2}$ & 600 & 2.2 & 389 & 8.0 & 68,89 \\
\hline 20-Cu & $\mathrm{CH}_{2} \mathrm{Cl}_{2}$ & 615 & 2.8 & 389 & 8.1 & 68 \\
\hline 20-CoPh & $\mathrm{CH}_{2} \mathrm{Cl}_{2}$ & 595 & 3.1 & 387 & 7.3 & 76 \\
\hline 20-Fe $\mathrm{C}_{6} \mathrm{H}_{5}$ & $\mathrm{CH}_{2} \mathrm{Cl}_{2}$ & 590 & 4.4 & 355 & 6.7 & 90 \\
\hline $20-\mathrm{Fe} \mathrm{C}_{6} \mathrm{~F}_{2} \mathrm{H}_{3}$ & $\mathrm{CH}_{2} \mathrm{Cl}_{2}$ & 593 & 4.2 & 383 & 6.8 & 90 \\
\hline $20-\mathrm{Fe} \mathrm{C}_{6} \mathrm{~F}_{3} \mathrm{H}_{2}$ & $\mathrm{CH}_{2} \mathrm{Cl}_{2}$ & 594 & 4.1 & 383 & 6.8 & 90 \\
\hline 20-Fe $\mathrm{C}_{6} \mathrm{~F}_{4} \mathrm{H}$ & $\mathrm{CH}_{2} \mathrm{Cl}_{2}$ & 623 & 3.5 & 378 & 7.9 & 90 \\
\hline 20-FeCl & $\mathrm{CH}_{2} \mathrm{Cl}_{2}$ & 617 & 3.7 & 368 & 8.8 & 90,91 \\
\hline 21-FeCl & $\mathrm{CH}_{2} \mathrm{Cl}_{2}$ & 687 & 2.8 & 365 & 4.9 & 91,92 \\
\hline 21-Co & $\mathrm{CH}_{2} \mathrm{Cl}_{2}$ & 659 & & 387 & & 79 \\
\hline 21-CoCl & $\mathrm{CH}_{2} \mathrm{Cl}_{2}$ & 604 & & 378 & & 79 \\
\hline \multirow[t]{3}{*}{$25-\mathrm{Ni}$} & $\mathrm{CH}_{2} \mathrm{Cl}_{2}$ & 643 & 5.6 & 393 & 9.2 & 13 \\
\hline & $n$-Hexane & 645 & & 394 & & 1 \\
\hline & 2-MTHF & 645 & & 393 & & 1 \\
\hline
\end{tabular}


115-Pd

116-Zn

116-Pd

117-Zn

117-Ni

117-Pd

118-Zn

118-Ni

122-Pd

122-Cu

168-Zn

170-Ni

171-Ni

171-Zn

173-Ni

173-Zn

176-Zn

177-Zn

178-Ni

178-Cu

181-Ni

183-Ni

186-Ni

188

189

190

191

192

193

194

195

196

197

198

199

200

201

\begin{tabular}{|c|c|c|c|c|c|}
\hline Acetonitrile & 658 & 5.5 & 392 & 8.3 & 13,62 \\
\hline Pyridine & 638 & 10.0 & 391 & 6.3 & 16 \\
\hline Pyridine & 642 & 10.0 & 392 & 17.0 & 16 \\
\hline $\mathrm{CH}_{2} \mathrm{Cl}_{2}$ & 644 & 4.1 & 390 & 8.9 & 18 \\
\hline $\mathrm{THF}$ & 655 & 5.2 & 395 & 7.9 & 93 \\
\hline $\mathrm{CH}_{2} \mathrm{Cl}_{2}$ & 606 & 6.2 & 390 & 12.3 & 18 \\
\hline $\mathrm{THF}$ & 616 & 6.7 & 388 & 11.0 & 93 \\
\hline $\mathrm{CH}_{2} \mathrm{Cl}_{2}$ & 631 & & 388 & & 23 \\
\hline $\mathrm{CHCl}_{3}$ & 638 & 7.9 & 391 & 17.4 & 36 \\
\hline $\mathrm{CHCl}_{3}$ & 606 & 5.0 & 389 & 8.7 & 36 \\
\hline $\mathrm{CHCl}_{3}$ & 638 & 4.1 & 391 & 7.9 & 36 \\
\hline $\mathrm{CHCl}_{3}$ & 624 & 3.5 & 391 & 8.5 & 36 \\
\hline $\mathrm{CHCl}_{3}$ & 618 & 5.6 & 390 & 12.0 & 37 \\
\hline $\mathrm{CHCl}_{3}$ & 596 & 3.3 & 388 & 11.2 & 37 \\
\hline $\mathrm{CHCl}_{3}$ & 590 & 3.0 & 390 & 6.9 & 37 \\
\hline $\mathrm{CHCl}_{3}$ & 719 & 4.8 & 409 & 5.6 & 38 \\
\hline $\mathrm{CHCl}_{3}$ & 691 & 6.8 & 408 & 8.1 & 38 \\
\hline Toluene & 632 & 4.9 & 395 & 8.5 & 94,95 \\
\hline Toluene & 644 & 4.8 & $\sim 395$ & $\sim 9$ & 95 \\
\hline $\mathrm{CH}_{2} \mathrm{Cl}_{2}$ & 629 & 0.3 & $406 / 426$ & $1.1 / 1.1$ & 12 \\
\hline $\mathrm{CH}_{2} \mathrm{Cl}_{2}$ & 739 & 2.9 & 359 & 4.2 & 89 \\
\hline $\mathrm{CH}_{2} \mathrm{Cl}_{2}$ & 630 & 5.7 & 429 & 17.5 & 5 \\
\hline Pyridine & 643 & 12.9 & 424 & 20.4 & 16 \\
\hline $\mathrm{CH}_{2} \mathrm{Cl}_{2}$ & 652 & 3.0 & 442 & 9.4 & 5 \\
\hline Pyridine & 652 & 15.5 & 445 & 3.6 & 16 \\
\hline Pyridine & 659 & 6.2 & 471 & 6.9 & 16 \\
\hline Pyridine & 685 & 15.3 & 507 & 47.6 & 16 \\
\hline $\mathrm{CHCl}_{3}$ & 701 & 4.6 & 409 & 6.0 & 65 \\
\hline $\mathrm{CHCl}_{3}$ & 723 & 7.0 & 409 & 11.3 & 96 \\
\hline $\mathrm{CHCl}_{3}$ & 690 & 5.1 & 407 & 7.3 & 65 \\
\hline $\mathrm{CH}_{2} \mathrm{Cl}_{2}$ & 717 & 2.3 & 385 & 2.9 & 64 \\
\hline $\mathrm{CH}_{2} \mathrm{Cl}_{2}$ & 817 & 2.2 & 406 & 6.8 & 89 \\
\hline $\mathrm{CH}_{2} \mathrm{Cl}_{2}$ & 643 & 3.5 & 416 & 13.0 & 89 \\
\hline $\mathrm{CH}_{2} \mathrm{Cl}_{2}$ & 630 & & 398 & & 89 \\
\hline $\mathrm{CH}_{2} \mathrm{Cl}_{2}$ & 630 & & 398 & & 89 \\
\hline $\mathrm{CH}_{2} \mathrm{Cl}_{2}$ & 609 & 3.2 & 390 & 12.3 & 89 \\
\hline $\mathrm{CH}_{2} \mathrm{Cl}_{2}$ & 608 & 4.4 & 390 & 14.0 & 89 \\
\hline $\mathrm{CH}_{2} \mathrm{Cl}_{2}$ & 624 & 4.9 & 396 & 7.9 & 97 \\
\hline $\mathrm{CH}_{2} \mathrm{Cl}_{2}$ & 620 & 2.5 & 395 & 4.6 & 97 \\
\hline $\mathrm{CH}_{2} \mathrm{Cl}_{2}$ & 624 & 6.5 & 417 & 8.5 & 97 \\
\hline $\mathrm{CH}_{2} \mathrm{Cl}_{2}$ & 628 & 2.3 & 407 & 3.4 & 97 \\
\hline $\mathrm{CH}_{2} \mathrm{Cl}_{2}$ & 628 & 4.0 & 412 & 5.8 & 97 \\
\hline $\mathrm{CH}_{2} \mathrm{Cl}_{2}$ & 656 & & 417 & & 12 \\
\hline $\mathrm{CH}_{2} \mathrm{Cl}_{2}$ & 694 & & 407 & & 12 \\
\hline $\mathrm{PhCN}$ & 620 & & 395 & & 68 \\
\hline $\mathrm{PhCN}$ & 628 & & 395 & & 68 \\
\hline
\end{tabular}




\begin{tabular}{|c|c|c|c|c|c|c|}
\hline 202 & $\mathrm{PhCN}$ & 625 & & 395 & & 68 \\
\hline 203 & $\mathrm{CH}_{2} \mathrm{Cl}_{2}$ & 860 & 0.9 & 426 & 2.2 & 68 \\
\hline 204 & $\mathrm{CH}_{2} \mathrm{Cl}_{2}$ & 872 & 0.85 & 427 & 2.0 & 68 \\
\hline 205 & $\mathrm{CH}_{2} \mathrm{Cl}_{2}$ & 870 & 0.59 & 426 & 1.7 & 68 \\
\hline 206 & $\mathrm{CH}_{2} \mathrm{Cl}_{2}$ & 862 & 0.81 & 423 & 2.2 & 68 \\
\hline 207 & $\mathrm{CH}_{2} \mathrm{Cl}_{2}$ & 875 & 1.4 & 423 & 3.0 & 68 \\
\hline 208 & $\mathrm{CH}_{2} \mathrm{Cl}_{2}$ & 877 & & 423 & & 68 \\
\hline 209-deoxy & $\mathrm{PB}^{\mathrm{e}}$ & 620 & & 375 & & 98 \\
\hline $209-\mathrm{O}_{2}$ & PB & 619 & & 387 & & 99 \\
\hline 209-CO & $\mathrm{PB}$ & 613 & & 391 & & 99 \\
\hline 210 & $\mathrm{CH}_{3} \mathrm{CN}$ & 625 & & 353 & & 100 \\
\hline 211 & $\mathrm{CH}_{3} \mathrm{CN}$ & 621 & & 346 & & 100 \\
\hline 212-deoxy & $\mathrm{PB}$ & 653 & & 386 & & 101 \\
\hline $212-\mathrm{O}_{2}$ & PB & 647 & & 380 & & 101 \\
\hline 212-CO & PB & 643 & & 393 & & 101 \\
\hline 213 & PB & 622 & & 385 & & 102 \\
\hline $214-\mathrm{N}_{3}{ }^{-}(1 \mathrm{MeIm}){ }^{\mathrm{e}}$ & DMSO & 624 & 5.0 & 388 & 10.3 & 103 \\
\hline 215 & $\mathrm{CH}_{2} \mathrm{Cl}_{2}$ & 632 & 5.6 & 367 & 10.0 & 6,104 \\
\hline 216 & $\mathrm{CH}_{2} \mathrm{Cl}_{2}$ & 628 & 8.9 & 379 & 12.3 & 6,104 \\
\hline 217 & $\mathrm{CH}_{2} \mathrm{Cl}_{2}$ & 632 & & 367 & & 104, 91 \\
\hline 218 & $\mathrm{CH}_{2} \mathrm{Cl}_{2}$ & 608 & & 390 & & 104,105 \\
\hline 219 & $\mathrm{CH}_{2} \mathrm{Cl}_{2}$ & 637 & 7.0 & 375 & 11.2 & 104 \\
\hline 220 & $\mathrm{CH}_{2} \mathrm{Cl}_{2}$ & 687 & 5.4 & 366 & 6.2 & 92,91 \\
\hline 221 & $\mathrm{CH}_{2} \mathrm{Cl}_{2}$ & 649 & 5.6 & 347 & 9.0 & 106 \\
\hline 222 & $\mathrm{CH}_{2} \mathrm{Cl}_{2}$ & 656 & 5.4 & 349 & 9.6 & 106 \\
\hline 223 & $\mathrm{CH}_{2} \mathrm{Cl}_{2}$ & 637 & 9.3 & 402 & 14.8 & 107 \\
\hline 224 & $\mathrm{CH}_{2} \mathrm{Cl}_{2}$ & 606 & 2.0 & 375 & 11.0 & 108 \\
\hline 225 & $\mathrm{CH}_{2} \mathrm{Cl}_{2}$ & 642 & 11.0 & 396 & 21.4 & 109 \\
\hline 226 & $\mathrm{CH}_{2} \mathrm{Cl}_{2}$ & 640 & 10.8 & 396 & 20.0 & 109 \\
\hline 228 & $\mathrm{CH}_{2} \mathrm{Cl}_{2}$ & 626 & 4.5 & 399 & 14.5 & 105 \\
\hline 229 & $\mathrm{CH}_{2} \mathrm{Cl}_{2}$ & 607 & 5.4 & 391 & 12.5 & 105 \\
\hline 230 & $\mathrm{CH}_{2} \mathrm{Cl}_{2}$ & 611 & 5.2 & 388 & 12.8 & 105 \\
\hline 231 & $\mathrm{CHCl}_{3}$ & $\sim 690$ & & 385 & & 110 \\
\hline 232 & $\mathrm{CH}_{2} \mathrm{Cl}_{2}$ & 672 & 1.2 & 373 & 4.5 & 111 \\
\hline 233 & $\mathrm{CHCl}_{3}$ & 612 & 2.9 & 385 & 5.7 & 112 \\
\hline 234 & $\mathrm{CHCl}_{3}$ & 600 & 3.3 & 384 & 5.5 & 112 \\
\hline 235 & $\mathrm{CHCl}_{3}$ & 625 & 4.0 & 356 & 5.7 & 112 \\
\hline
\end{tabular}

${ }^{\mathrm{a}}$ cpy $=p$-cyanopyridine; ${ }^{\mathrm{b}} \mathrm{dmap}=p$-dimethylaminopyridine $;{ }^{\mathrm{c}} \mathrm{pi}=$ pyrromellitic diimine bearing a pyridine ring at the $\mathrm{N}$-position, $\mathrm{Cl}_{2} \mathrm{Ph}, \mathrm{ClPh}, \mathrm{MePh}$ : differently substituted phthalimides; ${ }^{\mathrm{d}}$ phosphate buffer; ${ }^{\mathrm{e}}$ 1-methylimidazole. 
Table S3. Photophysical data for porphycenes: quantum yields of fluorescence $\left(\Phi_{\mathrm{F}}\right)$, triplet formation $\left(\Phi_{\mathrm{T}}\right)$, singlet oxygen generation $\left(\Phi_{\Delta}\right)$, and fluorescence decay time $\left(\tau_{\mathrm{F}}\right)$ (in $\left.\mathrm{ns}\right)$.

\begin{tabular}{|c|c|c|c|c|c|c|}
\hline & $\Phi_{\mathrm{F}}$ & $\tau_{\mathrm{F}}$ & $\Phi_{\mathrm{T}}$ & $\Phi_{\Delta}$ & Solvent & Reference \\
\hline \multirow[t]{12}{*}{1} & 0.36 & 10.2 & 0.42 & 0.30 & Toluene & \\
\hline & & 8.75 & & & $\mathrm{CH}_{2} \mathrm{Cl}_{2}$ & 2 \\
\hline & & 11.76 & & & Methanol & 2 \\
\hline & & & & 0.34 & Benzene & 113 \\
\hline & & 11.6 & & 0.32 & DPPC vesicles & 113 \\
\hline & 0.44 & & 0.3 & 0.34 & Benzene & 114 \\
\hline & 0.36 & 10.7 & & & $n$-Hexane & 115 \\
\hline & 0.42 & 12.0 & & & $\mathrm{CH}_{3} \mathrm{CN}$ & 115 \\
\hline & 0.49 & & & & DMSO & 115 \\
\hline & & 12.9 & & & $\mathrm{PVA}^{\mathrm{a}}$ & 115 \\
\hline & & 12.8 & & & $\mathrm{PVB}^{\mathrm{b}}$ & 115 \\
\hline & & & 0.40 & & $\mathrm{CH}_{3} \mathrm{CN}$ & 116 \\
\hline \multirow[t]{6}{*}{13} & 0.32 & 9.76 & & 0.35 & Toluene & 2 \\
\hline & & & & 0.36 & Benzene & 113 \\
\hline & & & & 0.37 & DPPC vesicles & 113 \\
\hline & 0.38 & & 0.4 & 0.36 & Benzene & 114 \\
\hline & 0.37 & 8.92 & 0.36 & 0.36 & Toluene & 21 \\
\hline & 0.38 & 9.9 & & & $\mathrm{THF}$ & 4 \\
\hline $13-\mathrm{SnF}_{2}$ & 0.139 & & & 0.46 & $\mathrm{CH}_{2} \mathrm{Cl}_{2}$ & 83 \\
\hline \multirow[t]{2}{*}{$13-\mathrm{SnCl}_{2}$} & 0.106 & & & 0.55 & $\mathrm{CH}_{2} \mathrm{Cl}_{2}$ & 83 \\
\hline & 0.092 & 3.4 & & & $\mathrm{CH}_{2} \mathrm{Cl}_{2}$ & 82 \\
\hline $13-\mathrm{SnBr}_{2}$ & 0.028 & & & 0.79 & $\mathrm{CH}_{2} \mathrm{Cl}_{2}$ & 83 \\
\hline 14 & 0.43 & 7.5 & & & $\mathrm{CH}_{2} \mathrm{Cl}_{2}$ & 5 \\
\hline \multirow[t]{2}{*}{19} & 0.02 & 5.0 & 0.04 & & Ethanol & 9 \\
\hline & 0.017 & & $<0.1$ & 0.06 & Toluene & 117 \\
\hline \multirow[t]{2}{*}{ 19-Zn } & 0.05 & 3.2 & & & Ethanol & 9 \\
\hline & & & & 0.68 & Toluene & 37 \\
\hline 19-Zn(py) & 0.03 & 3.3 & & & Toluene & 84 \\
\hline $19-\operatorname{Zn}(\mathbf{p i})^{c}$ & 0.003 & $0.14 / 3.9$ & & & Toluene & 84 \\
\hline $19-\mathrm{Zn}\left(\mathrm{PhCl}_{2}\right)^{\mathrm{c}}$ & 0.03 & 3.1 & & & Toluene & 84 \\
\hline $19-\mathrm{Zn}(\mathrm{PhCl})^{\mathrm{c}}$ & 0.03 & 3.3 & & & Toluene & 84 \\
\hline $19-\mathrm{Zn}(\mathrm{PhMe})^{\mathrm{c}}$ & 0.02 & 3.3 & & & Toluene & 84 \\
\hline $19-\mathrm{SnCl}_{2}$ & 0.125 & 2.68 & & & Toluene & 82 \\
\hline $19-\operatorname{Sn}(\mathrm{OH})_{2}$ & 0.094 & & & & Toluene & 88 \\
\hline $19-\operatorname{Sn}(\mathrm{FcCOO})_{2}$ & 0.01 & & & & Toluene & 88 \\
\hline 19-Pd & & & & 0.89 & Toluene & 37 \\
\hline \multirow[t]{6}{*}{23} & $2.9 \cdot 10^{-4}$ & & & & $n$-Hexane & 115 \\
\hline & $3.2 \cdot 10^{-4}$ & & & & $\mathrm{CH}_{3} \mathrm{CN}$ & 115 \\
\hline & $6.0 \cdot 10^{-4}$ & & & & $\mathrm{BuOH}$ & 115 \\
\hline & $1.5 \cdot 10^{-3}$ & & & & DMSO & 115 \\
\hline & & $0.003 / 0.017$ & & & $\mathrm{THF}$ & 118 \\
\hline & & $0.5 / 2.3$ & & & PVB & 115 \\
\hline
\end{tabular}




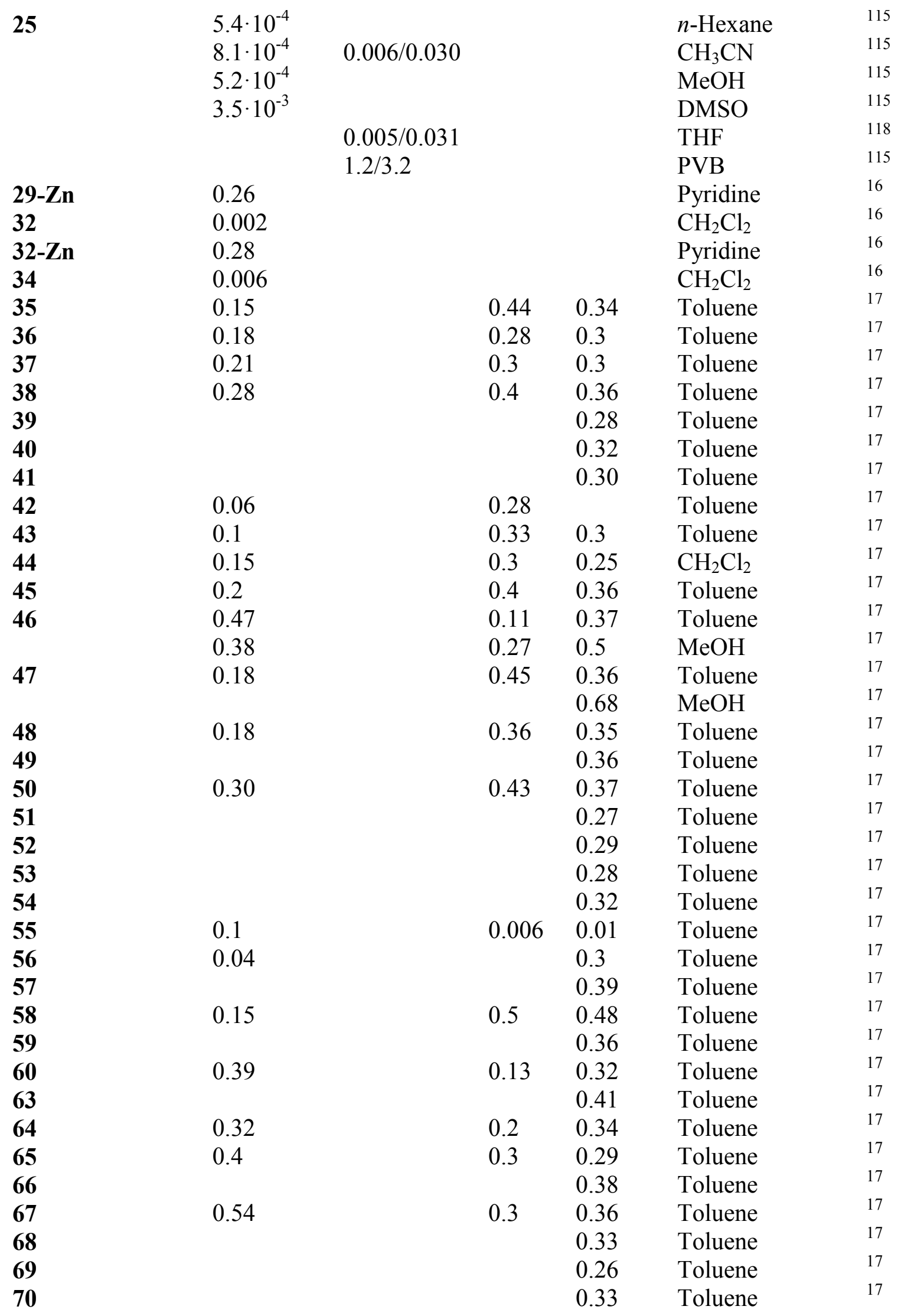




\begin{tabular}{|c|c|c|c|c|c|c|}
\hline 73 & 0.08 & 1.4 & 0.90 & 0.90 & Toluene & 21 \\
\hline 74 & 0.03 & 0.754 & 0.95 & 0.95 & Toluene & 21 \\
\hline 75 & 0.006 & 0.103 & 0.72 & 0.71 & Toluene & 21 \\
\hline 76 & 0.002 & 0.053 & 0.53 & 0.49 & Toluene & 21 \\
\hline \multirow[t]{2}{*}{77} & 0.33 & 8.38 & & & $\mathrm{CH}_{3} \mathrm{OD}$ & 24 \\
\hline & & & & 0.29 & $\mathrm{D}_{2} \mathrm{O}$ & 24 \\
\hline \multirow[t]{3}{*}{$78-79^{d}$} & 0.17 & 3.94 & & 0.25 & $\mathrm{CH}_{3} \mathrm{OD}$ & 24 \\
\hline & 0.10 & 1.61 & & & $\mathrm{H}_{2} \mathrm{O}$ & 24 \\
\hline & & & & 0.10 & $\mathrm{D}_{2} \mathrm{O}$ & 24 \\
\hline \multirow[t]{3}{*}{80} & $\approx 0$ & & & 0.01 & $\mathrm{CH}_{3} \mathrm{OD}$ & 24 \\
\hline & $\approx 0$ & & & & $\mathrm{H}_{2} \mathrm{O}$ & 24 \\
\hline & & & & $\approx 0$ & $\mathrm{D}_{2} \mathrm{O}$ & 24 \\
\hline \multirow[t]{2}{*}{81} & 0.14 & & & & $\mathrm{CH}_{2} \mathrm{Cl}_{2}$ & 26 \\
\hline & 0.11 & & & & $\begin{array}{l}\text { Sol-gel silica } \\
\text { plate }\end{array}$ & 26 \\
\hline \multirow[t]{3}{*}{83} & 0.20 & 7.8 & & & TMPA/TFSA $^{d}$ & 27 \\
\hline & 0.20 & 8.0 & & & $\mathrm{bmim} / \mathrm{TFSA}^{\mathrm{d}}$ & 27 \\
\hline & 0.18 & 7.0 & & & $\mathrm{CH}_{3} \mathrm{CN}$ & 27 \\
\hline 91 & 0.18 & 4.1 & & & $\mathrm{CH}_{2} \mathrm{Cl}_{2}$ & 30 \\
\hline 93 & 0.08 & & & & $\mathrm{CH}_{2} \mathrm{Cl}_{2}$ & 29 \\
\hline 94 & 0.02 & & & & $\mathrm{CH}_{2} \mathrm{Cl}_{2}$ & 29 \\
\hline 95 & 0.01 & & & & $\mathrm{CH}_{2} \mathrm{Cl}_{2}$ & 29 \\
\hline 96 & 0.006 & 0.14 & & & $\mathrm{CH}_{2} \mathrm{Cl}_{2}$ & 30 \\
\hline 97 & 0.007 & 0.19 & & & $\mathrm{CH}_{2} \mathrm{Cl}_{2}$ & 30 \\
\hline 117-Zn & 0.025 & 0.6 & & 0.12 & Toluene & 37 \\
\hline 117-Pd & & & & 0.73 & Toluene & 37 \\
\hline \multirow[t]{3}{*}{122} & 0.15 & 4.8 & 0.33 & 0.23 & Toluene & 95 \\
\hline & 0.14 & 3.6 & & & $\mathrm{THF}$ & 4 \\
\hline & 0.19 & 3.38 & & & $\mathrm{CH}_{2} \mathrm{Cl}_{2}$ & 47 \\
\hline 122-Pd & $<10^{-4}$ & & 0.78 & 0.78 & Toluene & 95 \\
\hline $122-\mathrm{Cu}$ & $<10^{-4}$ & & 0.35 & 0.24 & Toluene & 95 \\
\hline 123 & 0.22 & 4.3 & & 0.19 & Toluene & 43 \\
\hline 124 & 0.033 & 3.9 & & 0.16 & Toluene & 43 \\
\hline 125 & 0.004 & $0.81^{\mathrm{e}}$ & & 0.09 & Toluene & 43 \\
\hline 129 & 0.09 & 2.4 & & & $\mathrm{THF}$ & 4 \\
\hline 130 & 0.04 & 0.72 & & & $\mathrm{CH}_{2} \mathrm{Cl}_{2}$ & 47 \\
\hline 131 & 0.26 & 4.31 & & & $\mathrm{CH}_{2} \mathrm{Cl}_{2}$ & 47 \\
\hline 134 & 0.084 & 2.3 & & 0.10 & $\mathrm{THF}$ & 48 \\
\hline \multirow[t]{3}{*}{137} & 0.005 & 1.8 & & $0.03 / 0$ & $\mathrm{H}_{2} \mathrm{O}$ & 49 \\
\hline & 0.075 & 2.6 & & 004 & $\mathrm{MeOH}$ & 49 \\
\hline & & & & 0.193 & & 49 \\
\hline \multirow[t]{2}{*}{138} & 0.002 & 2.0 & & & 0.003 & 50,57 \\
\hline & 0.054 & 2.6 & & & 0.180 & 50 \\
\hline
\end{tabular}




\begin{tabular}{|c|c|c|c|c|c|c|}
\hline 142 & 0.07 & 0.8 & & 0.49 & Acetone & 52 \\
\hline 143 & 0.03 & 0.5 & & 0.30 & Acetone & 52 \\
\hline 144 & 0.08 & 0.6 & & 0.27 & Acetone & 52 \\
\hline 145 & 0.05 & 0.7 & & 0.24 & Acetone & 52 \\
\hline 146 & 0.05 & 0.9 & & 0.24 & Acetone & 52 \\
\hline 147 & 0.06 & 0.9 & & 0.30 & Acetone & 52 \\
\hline 148 & 0.04 & 0.6 & & 0.20 & Acetone & 52 \\
\hline 149 & 0.07 & 0.9 & & 0.31 & Acetone & 52 \\
\hline 150 & 0.23 & 3.91 & & & $\mathrm{CHCl}_{3}$ & 54 \\
\hline 151 & 0.21 & 3.68 & & & $\mathrm{CHCl}_{3}$ & 54 \\
\hline 152 & 0.21 & 3.59 & & & $\mathrm{CHCl}_{3}$ & 54 \\
\hline 153 & 0.18 & 3.01 & & & $\mathrm{CHCl}_{3}$ & 54 \\
\hline 154 & 0.16 & 2.95 & & & $\mathrm{CHCl}_{3}$ & 54 \\
\hline 155 & 0.18 & 3.06 & & & $\mathrm{CHCl}_{3}$ & 54 \\
\hline 158 & 0.03 & 0.52 & 0.045 & $\begin{array}{l}0.013 \\
0.023\end{array}$ & $\begin{array}{l}\text { Toluene } \\
\text { Benzene }\end{array}$ & $\begin{array}{l}58 \\
58\end{array}$ \\
\hline 159 & 0.02 & 0.34 & & 0.034 & $\begin{array}{l}\text { Toluene } \\
\text { Benzene }\end{array}$ & $\begin{array}{l}58 \\
58\end{array}$ \\
\hline 167 & & & & 0.55 & $\mathrm{CH}_{2} \mathrm{Cl}_{2} / \mathrm{MeOH}$ & 17 \\
\hline $\begin{array}{l}169,170 \\
170 \\
171\end{array}$ & $\begin{array}{l}<10^{-4} \\
0.42\end{array}$ & $\begin{array}{l}0.01-0.02 \\
6.0\end{array}$ & $<10^{-2}$ & $<10^{-4}$ & $\begin{array}{l}\text { Ar matrix } \\
\text { Toluene } \\
\mathrm{CH}_{2} \mathrm{Cl}_{2}\end{array}$ & $\begin{array}{l}119 \\
17 \\
5\end{array}$ \\
\hline 171-Zn & 0.31 & & & & Pyridine & 16 \\
\hline 173 & 0.32 & 6.9 & & & $\mathrm{CH}_{2} \mathrm{Cl}_{2}$ & 5 \\
\hline 173-Zn & 0.37 & & & & Pyridine & 16 \\
\hline 176 & 0.31 & & & & $\mathrm{CH}_{2} \mathrm{Cl}_{2}$ & 16 \\
\hline 176-Zn & 0.15 & & & & Pyridine & 16 \\
\hline 177-Zn & 0.17 & & & & Pyridine & 16 \\
\hline 186 & $<10^{-4}$ & & & $<10^{-3}$ & Toluene & 17 \\
\hline
\end{tabular}

${ }^{\mathrm{a}}$ poly(vinyl alcohol); ${ }^{\mathrm{b}}$ poly(vinyl butyral); ${ }^{\mathrm{c}}$ pi $=$ pyrromellitic diimine bearing a pyridine ring at the N-position, $\mathrm{Cl}_{2} \mathrm{Ph}, \mathrm{ClPh}, \mathrm{MePh}$ : differently substituted phthalimides; ${ }^{\mathrm{d}}$ average of two lifetimes; ${ }^{d}$ ionic liquid; ${ }^{e}$ the data reported for the mixture of two compounds. 


\section{References}

(1) Vogel, E.; Köcher, M.; Schmickler, H.; Lex, J. Porphycene - a Novel Porphin Isomer. Angew. Chem. Int. Ed. 1986, 25, 257-259.

(2) Aramendía, P. F.; Redmond, R. W.; Nonell, S.; Schuster, W.; Braslavsky, S. E.; Schaffner, K.; Vogel, E. The Photophysical Properties of Porphycenes: Potential Photodynamic Therapy Agents. Photochem. Photobiol. 1986, 44, 555-559.

(3) Vogel, E.; Balci, M.; Pramod, K.; Koch, P.; Lex, J.; Ermer, O. 2,7,12,17Tetrapropylporphycene - Counterpart of Octaethylporphyrin in the Porphycene Series. Angew. Chem. Int. Edit. 1987, 26, 928-931.

(4) Brenner, W.; Malig, J.; Oelsner, C.; Guldi, D. M.; Jux, N. Synthesis and PhysicoChemical Properties of Porphycenes. J. Porphyrins Phthalocyanines 2012, 16, 651-662.

(5) Kuzuhara, D.; Mack, J.; Yamada, H.; Okujima, T.; Ono, N.; Kobayashi, N. Synthesis, Structures, and Optical and Electrochemical Properties of Benzoporphycenes. Chem.-Eur. J. 2009, 15, 10060-10069.

(6) Lausmann, M.; Zimmer, I.; Lex, J.; Lueken, H.; Wieghardt, K.; Vogel, E. $\mu-$ Oxodiiron(III) Complexes of Porphycenes. Angew. Chem. Int. Ed. 1994, 33, 736-739.

(7) Czerski, I.; Listkowski, A.; Nawrocki, J.; Urbańska, N.; Piwoński, H.; Sokołowski, A.; Pietraszkiewicz, O.; Pietraszkiewicz, M.; Waluk, J. The Long and Winding Road to New Porphycenes. J. Porphyrins Phthalocyanines 2012, 16, 589-602.

(8) Vogel, E.; Koch, P.; Hou, X. L.; Lex, J.; Lausmann, M.; Kisters, M.; Aukauloo, M. A.; Richard, P.; Guilard, R. New Porphycene Ligands: Octaethyl- and Etioporphycene (OEPc and EtioPc)- Tetra-and Pentacoordinated Zinc Complexes of OEPc. Angew. Chem. Int. Ed. 1993, 32, 1600-1604.

(9) Berman, A.; Michaeli, A.; Feitelson, J.; Bowman, M. K.; Norris, J. R.; Levanon, H.; Vogel, E.; Koch, P. Photophysics and Photoinduced-Electron-Transfer Reactions of Zinc and Free-Base Octaethylporphycene. J. Phys. Chem. 1992, 96, 3041-3047.

(10) Guilard, R.; Aukauloo, M. A.; Tardieux, C.; Vogel, E. Synthesis of a New Alkylated Porphycene. Synthesis-Stuttgart 1995, 1480-1482.

(11) Hayashi, T.; Nakashima, Y.; Ito, K.; Ikegami, T.; Aritome, I.; Suzuki, A.; Hisaeda, Y. Synthesis, Structure, and Chemical Property of the First Fluorine-Containing Porphycene. Org. Lett. 2003, 5, 2845-2848.

(12) Masuno, M. N.; Robinson, B. C.; Phadke, A. S. A New Porphycene-Derived Ring Structure: Octaethylbenzochloracene. J. Porphyrins Phthalocyanines 2001, 5, 177-180.

(13) Vogel, E.; Köcher, M.; Lex, J.; Ermer, O. Steric Modulation of the Porphycene System by Alkyl Substituents: 9.10,19,20-Tetraalkylporphycenes. Isr. J. Chem. 1989, 29, 257266.

(14) Kuzuhara, D.; Yamada, H.; Yano, K.; Okujima, T.; Mori, S.; Uno, H. First Synthesis of Dodecasubstituted Porphycenes. Chem.--Eur. J. 2011, 17, 3376-3383.

(15) Ciąćka, P.; Fita, P.; Listkowski, A.; Kijak, M.; Nonell, S.; Kuzuhara, D.; Yamada, H.; Radzewicz, C.; Waluk, J. Tautomerism in Porphycenes: Analysis of Rate-Affecting Factors. J. Phys. Chem. B 2015, 119, 2292-2301.

(16) Kuzuhara, D.; Yamada, H.; Mori, S.; Okujima, T.; Uno, H. Synthesis, Structures and Properties of Benzoporphycenes and Naphthoporphycenes. J. Porphyrins Phthalocyanines 2011, 15, 930-942. 
(17) Braslavsky, S. E.; Müller, M.; Mártire, D. O.; Pörting, S.; Bertolotti, S. G.; Chakravorti, S.; Koç-Weier, G.; Knipp, B.; Schaffner, K. Photophysical Properties of Porphycene Derivatives (18 $\pi$ Porphyrinoids). J. Photochem. Photobiol. B: Biol. 1997, 40, 191-198. Erratum: 1998, 42, 79.

(18) Okawara, T.; Abe, M.; Shimakoshi, H.; Hisaeda, Y. Hydroxy-Functionalized Porphycenes: Structure, Spectroscopy, and Electrochemistry. Bull. Chem. Soc. Jpn. 2011, 84, 718-728.

(19) Richert, C.; Wessels, J. M.; Müller, M.; Kisters, M.; Benninghaus, T.; Goetz, A. E. Photodynamic Antitumor Agents: $\beta$-Methoxyethyl Groups Give Access to Functionalized Porphycenes and Enhance Cellular Uptake and Activity. J. Med. Chem. 1994, 37, 2797 2807.

(20) Polo, L.; Segalla, A.; Bertoloni, G.; Jori, G.; Schaffner, K.; Reddi, E. PolylysinePorphycene Conjugates as Efficient Photosensitizers for the Inactivation of Microbial Pathogens. J. Photochem. Photobiol. B: Biol. 2000, 59, 152-158.

(21) Shimakoshi, H.; Baba, T.; Iseki, Y.; Aritome, I.; Endo, A.; Adachi, C.; Hisaeda, Y. Photophysical and Photosensitizing Properties of Brominated Porphycenes. Chem. Commun. 2008, 2882-2884.

(22) Will, S.; Rahbar, A.; Schmickler, H.; Lex, J.; Vogel, E. Isocorroles - Novel Tetrapyrrolic Macrocycles. Angew. Chem. Int. Edit. 1990, 29, 1390-1393.

(23) Oertling, W. A.; Wu, W. S.; López-Garriga, J. J.; Kim, Y. Y.; Chang, C. K. Optical Absorptions and Raman-Scattering of Metalloporphycenes Reveal Electronic and Vibronic Properties Distinct from Those of Metalloporphyrins. J. Am. Chem. Soc. 1991, 113, 127-134.

(24) Baba, T.; Shimakoshi, H.; Endo, A.; Adachi, C.; Hisaeda, Y. Photophysical and Photocatalytic Properties of $\beta$-Sulfonatoporphycenes. Chem. Lett. 2008, 37, 264-265.

(26) Shimakoshi, H.; Baba, T.; Iseki, Y.; Endo, A.; Adachi, C.; Watanabe, M.; Hisaeda, Y. Photosensitizing Properties of the Porphycene Immobilized in Sol-Gel Derived Silica Coating Films. Tetrahedron Lett. 2008, 49, 6198-6201.

(27) Shimakoshi, H.; Sasaki, K.; Iseki, Y.; Hisaeda, Y. Synthesis and Photosensitizing Properties of Porphycene with Imidazolium Tag. J. Porphyrins Phthalocyanines 2012, 16, 530-536.

(28) Mak, N. K.; Kok, T. W.; Wong, R. N. S.; Lam, S. W.; Lau, Y. K.; Leung, W. N.; Cheung, N. H.; Huang, D. P.; Yeung, L. L.; Chang, C. K. Photodynamic Activities of Sulfonamide Derivatives of Porphycene on Nasopharyngeal Carcinoma Cells. J. Biomed. Sci. 2003, 10, 418-429.

(29) Okabe, T.; Kuzuhara, D.; Suzuki, M.; Aratani, N.; Yamada, H. Synthesis and Electrochemical Properties of Porphycene - Diketopyrrolopyrrole Conjugates. Org. Lett. 2014, 16, 3508-3511.

(30) Okabe, T.; Kuzuhara, D.; Aratani, N.; Yamada, H. Synthesis and Electronic Properties of Acetylene- and Butadiyne-Linked 3,3 '- Porphycene Dimers. J. Porphyrins Phthalocyanines 2014, 18, 849-855.

(31) Costa, R. D.; Malig, J.; Brenner, W.; Jux, N.; Guldi, D. M. Electron Accepting Porphycenes on Graphene. Adv. Mater. 2013, 25, 2600-2605. 
(32) Abe, M.; Yamada, H.; Okawara, T.; Fujitsuka, M.; Majima, T.; Hisaeda, Y. Covalently Attached Porphycene Ferrocene Dyads: Synthesis, Redox-Switched Emission, and Observation of the Charge-Separated State. Inorg. Chem. 2016, 55, 7-9.

(33) Haug, R.; Richert, C. A Porphycene-DNA Hybrid and Its DNA-Templated Interactions with a Porphyrin. J. Porphyrins Phthalocyanines 2012, 16, 545-555.

(34) Okawara, T.; Abe, M.; Hisaeda, Y. Synthesis of a Series of Multiply Meso-Acetoxylated Porphycenes. Tetrahedron Lett. 2014, 55, 6193-6197.

(35) Taneda, M.; Tanaka, A.; Shimakoshi, H.; Ikegami, A.; Hashimoto, K.; Abe, M.; Hisaeda, Y. Synthesis and Characterizations of Meso-Disubstituted Asymmetric Porphycenes. Tetrahedron Lett. 2013, 54, 5727-5729.

(36) Rana, A.; Panda, P. K. B-Tetrachlorotetramethoxyporphycenes: Positional Effect of Substituents on Structure and Photophysical Properties. Chem. Commun. 2015, 51, 12239-12242.

(37) Rana, A.; Panda, P. K. $\beta$-Octamethoxyporphycenes. Org. Lett. 2014, 16, 78-81.

(38) Rana, A.; Lee, S.; Kim, D.; Panda, P. K. $\beta$-Octakis(Methylthio)Porphycenes: Synthesis, Characterisation and Third Order Nonlinear Optical Studies. Chem. Commun. 2015, 51, 7705-7708.

(39) Chang, C. K.; Morrison, I.; Wu, W. S.; Chern, S. S.; Peng, S. M. Synthesis and Structure of N,N'-Bridged Porphycene. J Chem Soc Chem Comm 1995, 1173-1174.

(40) Setsune, J.; Hazama, K. Synthesis of N,N'-Etheno-Bridged Porphycene Hydroperchlorates. Tetrahedron Lett. 1997, 38, 2513-2516.

(41) Nonell, S.; Bou, N.; Borrell, J. I.; Teixidó, J.; Villanueva, A.; Juarranz, A.; Cañete, M. Synthesis of 2,7,12,17-Tetraphenylporphycene (TPPo). First Aryl-Substituted Porphycene for the Photodynamic Therapy of Tumors. Tetrahedron Lett. 1995, 36, 34053408.

(42) Berezin, D. B.; Talanova, A. E.; Shukhto, O. V.; Guseinov, S. S.; Krest'yaninov, M. A.; Petrova, D. V.; Semeikin, A. S. Synthesis and Physico-Chemical Properties of 2,7,12,17Tetraphenylporphycene and Its Metal Complexes. Russ. J. Gen. Chem. 2015, 85, 18761884.

(43) Arad, O.; Rubio, N.; Sánchez-García, D.; Borrell, J. I.; Nonell, S. Asymmetric Porphycenes: Synthesis and Photophysical Properties of 9-Substituted 2,7,12,17Tetraphenylporphycenes. J. Porphyrins Phthalocyanines 2009, 13, 376-381.

(44) Sánchez-García, D.; Borrell, J. I.; Nonell, S. One-Pot Synthesis of Substituted 2,2 'Bipyrroles. A Straightforward Route to Aryl Porphycenes. Org. Lett. 2009, 11, 77-79.

(45) Anguera, G.; Llinàs, M. C.; Batllori, X.; Sánchez-García, D. Aryl Nitroporphycenes and Derivatives: First Regioselective Synthesis of Dinitroporphycenes. J. Porphyrins Phthalocyanines 2011, 15, 865-870.

(46) Rosàs, E.; Santomá, P.; Hernandez, B.; Duran-Frigola, M.; Llinàs, M. C.; Ruiz-Gonzalez, R.; Nonell, S.; Sánchez-García, D.; Edelman, E. R.; Balcells, M. Modifications of Microvascular Ec Surface Modulate Phototoxicity of a Porphycene Anti-ICAM-1 Immunoconjugate; Therapeutic Implications. Langmuir 2013, 29, 9734-9743.

(47) Kuzuhara, D.; Nakaoka, H.; Okabe, T.; Aratani, N.; Yamada, H. Synthesis, Properties and Crystal Structures of 2,7,12,17-Tetraarylporphycenes. Heterocycles 2015, 90, 12141227. 
(48) García-Díaz, M.; Sánchez-García, D.; Soriano, J.; Sagristà, M. L.; Mora, M.; Villanueva, A.; Stockert, J. C.; Cañete, M.; Nonell, S. Temocene: The Porphycene Analogue of Temoporfin (Foscan (R)). Medchemcomm 2011, 2, 616-619.

(49) Ragàs, X.; Sánchez-García, D.; Ruiz-González, R.; Dai, T. H.; Agut, M.; Hamblin, M. R.; Nonell, S. Cationic Porphycenes as Potential Photosensitizers for Antimicrobial Photodynamic Therapy. J. Med. Chem. 2010, 53, 7796-7803.

(50) Ruiz-González, R.; Agut, M.; Reddi, E.; Nonell, S. A Comparative Study on Two Cationic Porphycenes: Photophysical and Antimicrobial Photoinactivation Evaluation. Int. J. Mol. Sci. 2015, 16, 27072-27086.

(51) Brenner, W.; Malig, J.; Costa, R. D.; Guldi, D. M.; Jux, N. Poly-Ortho-Functionalizable Tetraarylporphycene Platform-Synthesis of Octacationic Derivatives Towards the Layerby-Layer Design of Versatile Graphene Oxide Photoelectrodes. Adv. Mater. 2013, 25, 2314-2318.

(52) Planas, O.; Gallavardin, T.; Nonell, S. A Novel Fluoro-Chromogenic Click Reaction for the Labelling of Proteins and Nanoparticles with near-IR Theranostic Agents. Chem. Commun. 2015, 51, 5586-5589.

(53) Anju, K. S.; Ramakrishnan, S.; Thomas, A. P.; Suresh, E.; Srinivasan, A. 9,10,19,20Tetraarylporphycenes. Org. Lett. 2008, 10, 5545-5548.

(54) Ganapathi, E.; Chatterjee, T.; Ravikanth, M. Facile Synthesis of 9,10,19,20Tetraarylporphycenes. Eur. J. Org. Chem. 2014, 6701-6706.

(55) Brenner, W.; Jux, N. Dibenzoporphycene - Platform for the Generation of Fused Porphycenes. Eur. J. Org. Chem. 2015, 242-246.

(56) Sargent, A. L.; Hawkins, I. C.; Allen, W. E.; Liu, H.; Sessler, J. L.; Fowler, C. J. Global Versus Local Aromaticity in Porphyrinoid Macrocycles: Experimental and Theoretical Study of "Imidacene", an Imidazole-Containing Analogue of Porphycene. Chem. Eur. J. 2003, 9, 3065-3072.

(57) Nonell, S.; Borrell, J. I.; Borros, S.; Colominas, C.; Rey, O.; Rubio, N.; Sánchez-García, D.; Teixidó, J. 2,7,12,17-Tetra(P-Butylphenyl)-3,6,13,16-Tetraazaporphycene: The First Example of a Straightforward Synthetic Approach to a New Class of Photosensitizing Macrocycles. Eur. J. Org. Chem. 2003, 1635-1640.

(58) Rubio, N.; Sánchez-García, D.; Jiménez-Banzo, A.; Rey, Ó.; Borrell, J. I.; Teixidó, J.; Nonell, S. Effect of Aza Substitution on the Photophysical and Electrochemical Properties of Porphycenes: Characterization of the near-IR-Absorbing Photosensitizers 2,7,12,17-Tetrakis( $p$-Substituted Phenyl)-3,6,13,16-Tetraazaporphycenes. J. Phys. Chem. A 2006, 110, 3480-3487.

(59) De Munno, G.; Lucchesini, F.; Neidlein, R. 21,23-Dithiaporphycene - the First Aromatic Sulfur-Containing System with Porphycene Structure. Tetrahedron 1993, 49, 6863-6872.

(60) Nussbaumer, T.; Krieger, C.; Neidlein, R. 21,23-Dithia-3,13-Diazaporphycenes - Novel Aromatic Porphycene Analogues Incorporating Thiazole. Eur. J. Org. Chem. 2000, 24492457.

(61) Vogel, E.; Sicken, M.; Röhrig, P.; Schmickler, H.; Lex, J.; Ermer, O. Tetraoxaporphycene Dication. Angew. Chem. Int. Ed. 1988, 27, 411-414.

(62) Waluk, J.; Müller, M.; Swiderek, P.; Köcher, M.; Vogel, E.; Hohlneicher, G.; Michl, J. Electronic States of Porphycenes. J. Am. Chem. Soc. 1991, 113, 5511-5527.

(63) Dobkowski, J.; Galievsky, V.; Starukhin, A.; Vogel, E.; Waluk, J. Spectroscopy and Photophysics of Tetraalkyldibenzoporphycenes. J. Phys. Chem. A 1998, 102, 4966-4971. 
(64) Vargas-Zúñiga, G. I.; Roznyatovskiy, V. V.; Nepomnyaschii, A.; Lynch, V. M.; Sessler, J. L. $\pi$-Metal Complexes of $i$-Propyldinaphthoporphycene. J. Porphyrins

Phthalocyanines 2012, 16, 479-487.

(65) Sarma, T.; Panda, P. K.; Anusha, P. T.; Rao, S. V. Dinaphthoporphycenes: Synthesis and Nonlinear Optical Studies. Org. Lett. 2011, 13, 188-191.

(66) Roznyatovskiy, V.; Lynch, V.; Sessler, J. L. Dinaphthoporphycenes. Org. Lett. 2010, 12, 4424-4427.

(67) Oohora, K.; Ogawa, A.; Fukuda, T.; Onoda, A.; Hasegawa, J.; Hayashi, T. MesoDibenzoporphycene Has a Large Bathochromic Shift and a Porphycene Framework with an Unusual Cis Tautomeric Form. Angew. Chem. Int. Ed. 2015, 54, 6227-6230.

(68) Cuesta, L.; Karnas, E.; Lynch, V. M.; Chen, P.; Shen, J.; Kadish, K. M.; Ohkubo, K.; Fukuzumi, S.; Sessler, J. L. Metalloporphycenes: Synthesis and Characterization of (Pentamethylcyclopentadienyl)Ruthenium Sitting-Atop and $\pi$-Complexes. J. Am. Chem. Soc. 2009, 131, 13538-13547.

(69) Renner, M. W.; Forman, A.; Wu, W.; Chang, C. K.; Fajer, J. Electrochemical, Theoretical, and ESR Characterizations of Porphycenes. The $\pi$ Anion Radical of Nickel(II) Porphycene. J. Am. Chem. Soc. 1989, 111, 8618-8621.

(70) Guldi, D. M.; Neta, P.; Vogel, E. Radiolytic Reduction of Tetrapropylporphycene and Its Iron, Cobalt, Nickel, Copper, and Tin Complexes. J. Phys. Chem. 1996, 100, 4097-4103.

(71) Aoki, K.; Goshima, T.; Kozuka, Y.; Kawamori, Y.; Ono, N.; Hisaeda, Y.; Takagi, H. D.; Inamo, M. Electron Transfer Reaction of Porphyrin and Porphycene Complexes of $\mathrm{Cu}$ (II) and $\mathrm{Zn}(\mathrm{II})$ in Acetonitrile. Dalton Trans. 2009, 119-125.

(72) Toporowicz, M.; Ofir, H.; Levanon, H.; Vogel, E.; Köcher, M.; Pramod, K.; Fessenden, R. W. Triplet State of Metalloporphycenes: ZnPC1, PdPC2, PtPC2, and NiPC2.

Photochem. Photobiol. 1989, 50, 37-43.

(73) Che, C. M.; Cheung, K. K.; Li, Z. Y.; Wong, K. Y.; Wang, C. C.; Wang, Y. X-Ray Crystal-Structure of [2,7,12,17-Tetrapropylporphycenato]Platinum(II) - a Comparison of Nickel(II) and Platinum(II) Porphycene. Polyhedron 1994, 13, 2563-2567.

(74) Bernard, C.; Gisselbrecht, J. P.; Gross, M.; Vogel, E.; Lausmann, M. Redox Properties of Porphycenes and Metalloporphycenes. A Comparison with Porphyrins. Inorg. Chem. 1994, 33, 2393-2401.

(75) Shimakoshi, H.; Aritome, I.; Hirota, S.; Hisaeda, Y. Dioxygen Binding to a Cobalt(II) Porphycene Complex and Its Auto-Oxidized Cobalt(III) Complex. Bull. Chem. Soc. Jpn. 2005, 78, 1619-1623.

(76) Kadish, K. M.; Boulas, P. L.; Kisters, M.; Vogel, E.; Aukauloo, A. M.; D'Souza, F.; Guilard, R. Synthesis and Electrochemical Reactivity of $\sigma$-Bonded and N-Substituted Cobalt Porphycenes. Inorg. Chem. 1998, 37, 2693-2700.

(77) Hayashi, T.; Okazaki, K.; Shimakoshi, H.; Tani, F.; Naruta, Y.; Hisaeda, Y. Synthesis and Properties of Alkylperoxocobalt(III) Porphyrin and Porphycene. Chem. Lett. 2000, 90-91.

(78) Hayashi, T.; Okazaki, K.; Urakawa, N.; Shimakoshi, H.; Sessler, J. L.; Vogel, E.; Hisaeda, Y. Cobaltporphycenes as Catalysts. The Oxidation of Vinyl Ethers Via the Formation and Dissociation of Cobalt-Carbon Bonds. Organometallics 2001, 20, 30743078 . 
(79) Matsuo, T.; Komatsuzaki, K.; Tsuji, T.; Hayashi, T. Reaction of Cobalt Porphycene with Hydride Reagents: Spectroscopic Detection of Co-H Porphycene Species and Formation of Co-SnR 3 Porphycene Species. J. Porphyrins Phthalocyanines 2012, 16, 616-625.

(80) Okawara, T.; Abe, M.; Shimakoshi, H.; Hisaeda, Y. Redox Gradations in Ruthenium Porphycene Complexes and the Porphyrin Analogs: Axial and Macrocyclic Ligand Effects. Chem. Lett. 2008, 37, 906-907.

(81) Okawara, T.; Abe, M.; Ashigara, S.; Hisaeda, Y. Molecular Structures, Redox Properties, and Photosubstitution of Ruthenium(II) Carbonyl Complexes of Porphycene. $J$. Porphyrins Phthalocyanines 2015, 19, 233-241.

(82) Maeda, D.; Shimakoshi, H.; Abe, M.; Hisaeda, Y. Syntheses and Photophysical Behavior of Porphyrin Isomer Sn(IV) Complexes. Inorg. Chem. 2009, 48, 9853-9860.

(83) Taneda, M.; Maeda, D.; Shimakoshi, H.; Abe, M.; Hisaeda, Y. Preparations and Photosensitizing Properties of 2,7,12,17-Tetra-N-Propylporphycenatotin(IV) Dihalide Complexes. Bull. Chem. Soc. Jpn. 2010, 83, 667-671.

(84) Fujitsuka, M.; Shimakoshi, H.; Tojo, S.; Cheng, L. L.; Maeda, D.; Hisaeda, Y.; Majima, T. Electron Transfer in the Supramolecular Donor-Acceptor Dyad of Zinc Porphycene. $J$. Phys. Chem. A 2009, 113, 3330-3335.

(85) D'Souza, F.; Boulas, P.; Aukauloo, A. M.; Guilard, R.; Kisters, M.; Vogel, E.; Kadish, K. M. Electrochemical, UV/Visible, and EPR Characterization of Metalloporphycenes Containing First-Row Transition-Metals. J. Phys. Chem. 1994, 98, 11885-11891.

(86) Fowler, C. J.; Sessler, J. L.; Lynch, V. M.; Waluk, J.; Gebauer, A.; Lex, J.; Heger, A.; Zuniga-y-Rivero, F.; Vogel, E. Metal Complexes of Porphycene, Corrphycene, and Hemiporphycene: Stability and Coordination Chemistry. Chem.-Eur. J. 2002, 8, 34853496.

(87) Maeda, D.; Shimakoshi, H.; Abe, M.; Hisaeda, Y. Synthesis and Photochemical Properties of a New Molybdenum Porphycene Complex. Dalton Trans. 2009, 140-145.

(88) Maeda, D.; Shimakoshi, H.; Abe, M.; Fujitsuka, M.; Majima, T.; Hisaeda, Y. Synthesis of a Novel Sn(IV) Porphycene-Ferrocene Triad Linked by Axial Coordination and Solvent Polarity Effect in Photoinduced Charge Separation Process. Inorg. Chem. 2010, 49, 2872-2880.

(89) D'Souza, F.; Boulas, P. L.; Kisters, M.; Sambrotta, L.; Aukauloo, A. M.; Guilard, R.; Kadish, K. M. Effect of Peripheral Substitution and Extended Conjugation on the Redox Potentials of Nickel Porphycenes. Inorg. Chem. 1996, 35, 5743-5746.

(90) Kadish, K. M.; Tabard, A.; Van Caemelbecke, E.; Aukauloo, A. M.; Richard, P.; Guilard, R. Physicochemical Characterization of Sigma-Bonded Aryl Iron(III) Porphycenes. XRay Structures of (EtioPc)Fe(3,5- $\left.\mathrm{C}_{6} \mathrm{~F}_{2} \mathrm{H}_{3}\right)$ and (EtioPc) $\operatorname{In}\left(\mathrm{C}_{6} \mathrm{H}_{5}\right)$, where EtioPc Is the Dianion of 2,7,12,17-Tetraethyl-3,6,13,16-Tetramethylporphycene. Inorg. Chem. 1998, 37, 6168-6175.

(91) Ito, K.; Matsuo, T.; Aritome, I.; Hisaeda, Y.; Hayashi, T. Isolable Iron(II)-Porphycene Derivative Stabilized by Introduction of Trifluoromethyl Groups on the Ligand Framework. Bull. Chem. Soc. Jpn. 2008, 81, 76-83.

(92) Hayashi, T.; Nakashima, Y.; Ito, K.; Ikegami, T.; Aritome, I.; Aoyagi, K.; Ando, T.; Hisaeda, Y. Synthesis, Characterization, and Autoreduction of a Highly ElectronDeficient Porphycenatoiron(III) with Trifluoromethyl Substituents. Inorg. Chem. 2003, 42, 7345-7347. 
(93) Okawara, T.; Abe, M.; Shimakoshi, H.; Hisaeda, Y. A Pd(II)-Hydroxyporphycene: Synthesis, Characterization, and Photoinduced Proton-Coupled Electron Transfer. Res Chem Intermediat 2013, 39, 161-176.

(94) Cañete, M.; Ortiz, A.; Juarranz, A.; Villanueva, A.; Nonell, S.; Borrell, J. I.; Teixidó, J.; Stockert, J. C. Photosensitizing Properties of Palladium-Tetraphenylporphycene on Cultured Tumour Cells. Anti-Cancer Drug Des. 2000, 15, 143-150.

(95) Rubio, N.; Prat, F.; Bou, N.; Borrell, J. I.; Teixidó, J.; Villanueva, A.; Juarranz, A.; Cañete, M.; Stockert, J. C.; Nonell, S. A Comparison between the Photophysical and Photosensitising Properties of Tetraphenyl Porphycenes and Porphyrins. New J. Chem. 2005, 29, 378-384.

(96) Sarma, T.; Panda, P. K. Effect of $\beta-\beta^{\prime}$ Fusion on Metal Ion Complexation of Porphycene. J. Chem. Sci. 2015, 127, 235-240.

(97) Feihl, S.; Costa, R. D.; Brenner, W.; Margraf, J. T.; Casillas, R.; Langmar, O.; Browa, A.; Shubina, T. E.; Clark, T.; Jux, N.et al. Integrating Metalloporphycenes into p-Type NiOBased Dye-Sensitized Solar Cells. Chem. Commun. 2014, 50, 11339-11342.

(98) Hayashi, T.; Dejima, H.; Matsuo, T.; Sato, H.; Murata, D.; Hisaeda, Y. Blue Myoglobin Reconstituted with an Iron Porphycene Shows Extremely High Oxygen Affinity. J. Am. Chem. Soc. 2002, 124, 11226-11227.

(99) Matsuo, T.; Dejima, H.; Hirota, S.; Murata, D.; Sato, H.; Ikegami, T.; Hori, H.; Hisaeda, Y.; Hayashi, T. Ligand Binding Properties of Myoglobin Reconstituted with Iron Porphycene: Unusual $\mathrm{O}_{2}$ Binding Selectivity against Co Binding. J. Am. Chem. Soc. 2004, 126, 16007-16017.

(100) Oohora, K.; Kihira, Y.; Mizohata, E.; Inoue, T.; Hayashi, T. C( sp $\left.^{3}\right)-H$ Bond Hydroxylation Catalyzed by Myoglobin Reconstituted with Manganese Porphycene. $J$. Am. Chem. Soc. 2013, 135, 17282-17285.

(101) Matsuo, T.; Ito, K.; Nakashima, Y.; Hisaeda, Y.; Hayashi, T. Effect of Peripheral Trifluoromethyl Groups in Artificial Iron Porphycene Cofactor on Ligand Binding Properties of Myoglobin. J. Inorg. Biochem. 2008, 102, 166-173.

(102) Matsuo, T.; Ikegami, T.; Sato, H.; Hisaeda, Y.; Hayashi, T. Ligand Binding Properties of Two Kinds of Reconstituted Myoglobins with Iron Porphycene Having Propionates: Effect of $\beta$-Pyrrolic Position of Two Propionate Side Chains in Porphycene Framework. J. Inorg. Biochem. 2006, 100, 1265-1271.

(103) Neya, S.; Chang, C. K.; Okuno, D.; Hoshino, T.; Hata, M.; Funasaki, N. Control of Iron(III) Spin-State in the Model Complexes of Azide Hemoprotein by Porphycene, Corrphycene, and Hemiporphycene Macrocycles. Inorg. Chem. 2005, 44, 1193-1195.

(104) Kadish, K. M.; Boulas, P.; Dsouza, F.; Aukauloo, M. A.; Guilard, R.; Lausmann, M.; Vogel, E. Electrode-Reactions of $\mu$-Oxo Iron(III) Porphycene Dimers - Formation of Stable $\left[[(\mathrm{Pc}) \mathrm{Fe}]_{2} \mathrm{O}\right]^{n}$ Complexes where $\mathrm{n}=-4$ to +4 . Inorg. Chem. 1994, 33, 471-476.

(105) Guilard, R.; Pichon-Pesme, V.; Lachekar, H.; Lecomte, C.; Aukauloo, A. M.; Boulas, P. L.; Kadish, K. M. Synthesis and Electrochemistry of Aluminum Porphycenes. Crystal and Molecular Structure of Methyl- $\sigma$-Bonded Aluminum Etioporphycene. J. Porphyrins Phthalocyanines 1997, 1, 109-119.

(106) Baba, T.; Shimakoshi, H.; Aritome, I.; Hisaeda, Y. Synthesis and Characterization of $\mu-$ Oxodiiron(III) Complexes of Porphycenes with Electron-Withdrawing Substituents. Chem. Lett. 2004, 33, 906-907. 
(107) Kadish, K. M.; D'Souza, F.; Van Caemelbecke, E.; Boulas, P.; Vogel, E.; Aukauloo, A. M.; Guilard, R. Electrochemistry of New $\sigma$-Bonded Metal(III) Complexes with Tetrapyrrole Ligands. Reactions of $($ EtioPc $) \mathrm{M}\left(\mathrm{C}_{6} \mathrm{H}_{5}\right)$ and EtioPc $) \mathrm{FeCl}$ where $\mathrm{M}=\mathrm{Fe}$ or In and EtioPc Is the Dianion of 2,7,12,17-Tetraethyl-3,6,13,16-Tetramethylporphycene. Inorg. Chem. 1994, 33, 4474-4479.

(108) Abe, M.; Futagawa, H.; Ono, T.; Yamada, T.; Kimizuka, N.; Hisaeda, Y. An Electropolymerized Crystalline Film Incorporating Axially-Bound Metalloporphycenes: Remarkable Reversibility, Reproducibility, and Coloration Efficiency of Ruthenium(II/III)-Based Electrochromism. Inorg. Chem. 2015, 54, 11061-11063.

(109) Stępień, M.; Donnio, B.; Sessler, J. L. Discotic Liquid-Crystalline Materials Based on Porphycenes: A Mesogenic Metalloporphycene-Tetracyanoquinodimethane (TCNQ) Adduct. Chem.-Eur. J. 2007, 13, 6853-6863.

(110) Che, C. M.; Li, Z. Y.; Guo, C. X.; Wong, K. Y.; Chern, S. S.; Peng, S. M. Synthesis and Crystal-Structure of $(2,7,12,17$-Tetrapropylporphycenato)bis-[Tricarbonylrhenium(I)]. Inorg. Chem. 1995, 34, 984-987.

(111) Zhang, W. N.; Chang, Y.; Wu, F.; Mack, J.; Kobayashi, N.; Shen, Z. Synthesis, Structure and Spectroscopic Properties of a Porphycene-Re ${ }^{\mathrm{I}}$ Complex. J. Porphyrins Phthalocyanines 2011, 15, 622-631.

(112) Li, Z. Y.; Huang, J. S.; Che, C. M.; Chang, C. K. Unusual Metalloporphycenes. First Syntheses of Carbonyl-Containing and Dioxo-Containing Osmium and Ruthenium Tetrapropylporphycene Complexes. Inorg. Chem. 1992, 31, 2670-2672.

(113) Redmond, R. W.; Valduga, G.; Nonell, S.; Braslavsky, S. E.; Schaffner, K.; Vogel, E.; Pramod, K.; Köcher, M. The Photophysical Properties of Porphycene Incorporated in Small Unilamellar Lipid Vesicles. J. Photochem. Photobiol. B: Biol. 1989, 3, 193-207.

(114) Nonell, S.; Aramendía, P. F.; Heihoff, K.; Negri, R. M.; Braslavsky, S. E. Laser-Induced Optoacoustics Combined with near-Infrared Emission. An Alternative Approach for the Determination of Intersystem Crossing Quantum Yields Applied to Porphycenes. J. Phys. Chem. 1990, 94, 5879.

(115) Gil, M.; Dobkowski, J.; Wiosna-Sałyga, G.; Urbańska, N.; Fita, P.; Radzewicz, C.; Pietraszkiewicz, M.; Borowicz, P.; Marks, D.; Glasbeek, M.et al. Unusual, Solvent Viscosity-Controlled Tautomerism and Photophysics: Meso-Alkylated Porphycenes. $J$. Am. Chem. Soc. 2010, 132, 13472-13485.

(116) Lament, B.; Karpiuk, J.; Waluk, J. Determination of Triplet Formation Efficiency from Kinetic Profiles of the Ground State Recovery. Photochem. Photobiol. Sci. 2003, 2, $267-$ 272.

(117) Vogel, E.; Bröring, M.; Weghorn, S. J.; Scholz, P.; Deponte, R.; Lex, J.; Schmickler, H.; Schaffner, K.; Braslavsky, S. E.; Müller, M.et al. Octaethylhemiporphycene: Synthesis, Molecular Structure, and Photophysics. Angew. Chem. Int. Edit. 1997, 36, 1651-1654.

(118) Gil, M.; Organero, J. A.; Waluk, J.; Douhal, A. Ultrafast Dynamics of Alkyl-Substituted Porphycenes in Solution. Chem. Phys. Lett. 2006, 422, 142-146.

(119) Dobkowski, J.; Lobko, Y.; Gawinkowski, S.; Waluk, J. Energy Relaxation Paths in Matrix-Isolated Excited Molecules: Comparison of Porphycene with Dibenzoporphycenes. Chem. Phys. Lett. 2005, 128-132.

(120) Gisselbrecht, J. P.; Gross, M.; Köcher, M.; Lausmann, M.; Vogel, E. Redox Properties of Porphycenes and Metalloporphycenes as Compared with Porphyrins. J. Am. Chem. Soc. 1990, 112, 8618-8620. 